\title{
The quantitative impact of placements on allied health time use and productivity in healthcare facilities: A systematic review with meta-analysis
}

\author{
E. Bourne ${ }^{1}$, K. Short ${ }^{3}$, L. McAllister ${ }^{1}$ \& S. Nagarajan ${ }^{2}$
}

\begin{abstract}
Introduction: The current and future health needs of the population pose challenges for healthcare services, which face increased pressure for service provision, and for universities educating graduates to meet this clinical demand. One aspect influencing allied health $(\mathrm{AH})$ clinician willingness to offer student placements is the perceptions of impact on patient activity levels and clinician time. This systematic review synthesises the evidence quantifying student impact on $\mathrm{AH}$ patient activity, clinician time and productivity.
\end{abstract}

Methods: Searches of peer-reviewed literature published since 1990 were conducted in Medline, CINAHL, Scopus and EMBASE and supplemented by other sources. Selected studies reported clinician-recorded patient activity and/or time participating in services provided by nutrition and dietetics, occupational therapy, physiotherapy and speech pathology, with and without students present. Studies meeting eligibility criteria $(n=23)$ were rated using the McMaster Guidelines for Critical Review Form: Quantitative Studies. Effect size calculations and meta-analysis were planned if sufficient studies reported similar outcome measures.

Results: Seventeen studies contributed to four meta-analyses: patient activity levels, direct clinical time, clinical billed units and direct time per patient. Pooled results were neutral or favoured increases in activity or time during student placements. Methodological variation and research quality inhibited more comprehensive analysis.

1 University of Sydney, Work Integrated Learning, Faculty of Health Sciences

2 University of Sydney, Northern Clinical School, Faculty of Medicine and Health

3 Liverpool Hospital, South Western Sydney Local Health District

Correspondence

Elizabeth Bourne

University of Sydney

Work Integrated Learning

Faculty of Health Sciences

75 East St

Lidcombe

Sydney

Australia

Tel: +61 290367412

Email: elizabeth.bourne@sydney.edu.au 
Conclusions: Data showed students may have a neutral or positive effect on AH patient activity levels and clinical time. Potential factors influencing data are discussed, and the areas of focus for further research are suggested.

Keywords: allied health; clinical education; meta-analysis; productivity; student placement; systematic review; time use.

\section{Introduction}

The need for a larger health workforce has been predicted in countries such as Australia (National Health Workforce Taskforce, 2009) and the United States (United States Department of Labor, 2018), and a resulting growth in enrolments in AH professional preparation courses has occurred (Evenson, Roberts, Kaldenberg, Barnes, \& Ozelie, 2015; HWA, 2014). Within these courses, an important contributor to student clinical competency is practical workplace experience (Rodger et al., 2008). AH university students spend 25\% (Dean, Alam, \& Refshauge, 2010) to 33\% (Hall, Poth, Manns, \& Beaupre, 2015) of their education completing these experiences. In this paper, these will be termed placements, encompassing all similar terms, such as practicum or fieldwork. Internationally, universities have faced challenges to secure sufficient placements in $\mathrm{AH}$ for a number of years (Hall et al., 2015; Huddleston, 1999; McAllister, 2005). With the increased placement numbers required, there is mounting pressure on healthcare services that already provide many placements (Bowles et al., 2014; Roberts, Evenson, Kaldenberg, Barnes, \& Ozelie, 2015). Thus, the situation for students, universities and placement providers (who are also future employers) requires urgent attention and creative action to ensure a supply of sufficiently well-trained health professionals.

The impact of students on the time use of a clinician — the placement supervisor-has been suggested as a key consideration for AH before undertaking student supervision (e.g., Davies, Hanna, \& Cott, 2011; Evenson et al., 2015) and a barrier for their employers (O'Brien et al., 2017). Clinician time use, with or without students, has been variably categorised, however commonly includes direct clinical time (activities in the presence of the patient or client, hereafter referred to as patient), indirect clinical time (activities related to the patient and needed for their care, e.g., documentation and interaction with other professionals) and non-clinical activities (such as administration, professional development, staff supervision, quality improvement and research). Time spent teaching significant others, including other professionals and/or students, has also been recorded. Some studies have found that clinicians are concerned that supervising students reduces opportunities for the completion of non-clinical tasks (e.g., Sevenhuysen \& Haines, 2011). Many clinicians also believe that students reduce their patient activity levels (that is, the number of direct patient services per day, e.g., occasions of service) and/or available clinical time, resulting in lower productivity, that is, they see less patients per hour (e.g., Hall et al., 2015). Investigating productivity is important even when clinical time is increased with students present, as it can establish whether students see more patients with the extra clinical time, take longer to see the same number of patients or see less patients but take more time than a clinician without students. 
This paper addresses the question: Does supervising students quantitatively impact clinicians' patient activity, time use and productivity in the AH professions of nutrition and dietetics, occupational therapy, physiotherapy and speech pathology? These four professions were chosen for the similiarity of their more person-oriented rather than technique-oriented work (Campbell, Eley, \& McAllister, 2014). Clinician time spent with patients and on other activities has been similarly categorised across these professions, and quantitative data combining some professions has previously been reported (e.g., Rodger et al., 2012). Phelan, Daniels and Hewitt (1999) summarised the literature reporting costs, benefits and productivity of student placements for $\mathrm{AH}$ from 1976-1998, however no more recent reviews were located. This paper analyses key findings in studies investigating patient activity, clinical and non-clinical time use and productivity of AH clinicians, with and without students, since 1990 and points to areas where further research is needed. The specific objectives of this review were to research within the professions of nutrition and dietetics, occupational therapy, physiotherapy and speech pathology in order to:

A. compare patient activity levels using quantitative data during periods when clinicians are supervising students and when they are not

B. profile and compare clinicians' time use using quantitative data during periods with and without students

C. explore quantitative links between time and activity data and establish the productivity impact of student placements.

\section{Methods}

This quantitative systematic review was designed based on the PRISMA guidelines (Moher, Liberati, Tetzlaff, Altman, \& The, 2009).

\section{Information sources}

A comprehensive search was conducted from January 1990 to August 2017. Electronic searches of Medline, CINAHL, Scopus and Embase databases used the search terms outlined in the example in Table 1. Databases and final search terms were selected for their potential wide coverage of the research topic within the identified professions across published articles and grey literature based on advice from a professional librarian. Following this process, hand searches of reference lists of selected articles were completed and supplemented by authors' recommendations of other relevant studies not identified using the above methods.

\section{Eligibility criteria}

Inclusion/exclusion criteria were agreed upon by all authors prior to commencement (see Table 2). Only papers with quantitative reporting of clinician-recorded patient activity and/or time in the identified professions were included in the final analysis. Similar contexts were sought through restriction of placement setting, geographical location and publication date to enable meaningful data pooling and interpretation. 


\section{IMPACT OF PLACEMENTS ON ALLIED HEALTH TIME USE AND PRODUCTIVITY}

Table 1

Search Strategy Utilized in Medline database++

\begin{tabular}{|c|c|c|}
\hline Professions (Population) & $\begin{array}{l}\text { Supervising Students } \\
\text { (Intervention/Exposure) }\end{array}$ & $\begin{array}{l}\text { Activity, Time Use \& Productivity } \\
\text { (Outcome) }\end{array}$ \\
\hline $\begin{array}{l}\text { Physical Therapy Specialty/ or } \\
\text { physical therap*.mp. }\end{array}$ & clinical educat*.mp\#. & $\begin{array}{l}\text { efficiency/ or "time and motion } \\
\text { studies"/ or time management/ }\end{array}$ \\
\hline physiotherap*.mp. & fieldwork* ${ }^{*} . m p$ & productivity.mp. \\
\hline $\begin{array}{l}\text { Occupational Therapy/ or occupational } \\
\text { therap*.mp. }\end{array}$ & workplace learning.mp. & productivities.mp. \\
\hline (speech adj2 path*).mp. & workplace training.mp. & clinical time.mp. \\
\hline $\begin{array}{l}\text { Speech-Language Pathology/ or speech } \\
\text { language patholog*.mp }\end{array}$ & clinical placement*.mp. & (clinical adj1 time).mp. \\
\hline Speech Therapy/ or speech therap*.mp. & student placement* & $\begin{array}{l}\text { health services/ or community health } \\
\text { services/ or dietary services/ or student } \\
\text { health services/ or health services } \\
\text { administration/ }\end{array}$ \\
\hline speech language therap*.mp. & field placement ${ }^{*} . \mathrm{mp}$. & health service ${ }^{*} . \mathrm{mp}$. \\
\hline Dietetics/ & clinical instruct*.mp. & Workload/ \\
\hline dietitian*.mp. & clinical coordination.mp. & workload* $^{*}$ mp. \\
\hline \multirow[t]{12}{*}{ (nutrition and dietetics).mp. } & fieldwork coordination.mp. & workflow/ \\
\hline & Preceptorship/ & workflow*.mp. \\
\hline & preceptor* $^{*}$ & Time Factors/ \\
\hline & Students/ & time usage.mp. \\
\hline & student supervisor* ${ }^{*} . \mathrm{mp}$ & $\begin{array}{l}\text { health expenditures/ or health } \\
\text { resources/ or "health services needs } \\
\text { and demand"/ }\end{array}$ \\
\hline & student supervision.mp. & health care delivery*.mp \\
\hline & clinical coordinator*.mp. & health care cost* \\
\hline & fieldwork coordinator*.mp. & health service deliver*.mp. \\
\hline & clinical teach*.mp. & (health adj1 service).mp. \\
\hline & (clinical adj1 teach*).mp. & work time.mp. \\
\hline & practicum.mp. & work/ or work performance/ \\
\hline & & task performance \\
\hline
\end{tabular}

\section{Notes:}

++ Within column terms were combined using the Boolean operator "OR" and across columns "AND" was used to group searches together $\# \mathrm{mp}$ = title, abstract, original title, name of substance word, subject heading word, keyword heading word, protocol supplementary concept word, rare disease supplementary concept word, unique identifier, synonyms

${ }^{*}$ = truncation, searching for word endings, e.g., clinical educat* searches clinical educator, clinical educators, clinical education, etc 
Table 2

Inclusion and Exclusion Criteria

\begin{tabular}{|c|c|c|}
\hline Criterion & Inclusion & Exclusion \\
\hline 1. Professions & $\begin{array}{l}\text { Studies involved dietitians, } \\
\text { occupational therapists, physiotherapists } \\
\text { or speech-language pathologists and } \\
\text { students from the same profession }\end{array}$ & $\begin{array}{l}\text { - Clinicians from medicine, nursing or } \\
\text { other allied health professions } \\
\text { - Supervision of students from } \\
\text { another profession }\end{array}$ \\
\hline $\begin{array}{l}\text { 2. Data collection method } \\
\text { and reporting detail }\end{array}$ & $\begin{array}{l}\text { Clinician recorded time and/or patient } \\
\text { activity quantitatively reported }\end{array}$ & $\begin{array}{l}\text { - Data recorded by students only } \\
\text { - Qualitative report of activity or time } \\
\text { - Full text with numerical data } \\
\text { unavailable or insufficient (e.g. } \\
\text { percentages only) \# }\end{array}$ \\
\hline 3. Data collection period & $\begin{array}{l}\text { Included collection of the above data in } \\
\text { periods with and without students } \\
\text { present }\end{array}$ & $\begin{array}{l}\text { No collection or reporting of data both } \\
\text { with and without students }\end{array}$ \\
\hline 4. Placement setting & $\begin{array}{l}\text { Predominantly recorded in public } \\
\text { in healthcare facilities (Search 1) } \\
\text { Appeared to be predominantly recorded } \\
\text { in healthcare facilities providing patient } \\
\text { care (e.g., hospital or outpatient clinic) } \\
\text { (Search 2) \# }\end{array}$ & $\begin{array}{l}\text { Primarily in other setting, e.g., private } \\
\text { healthcare facilities or school (Search 1) } \\
\text { Primarily appeared to be in other setting, } \\
\text { e.g., school, residential facilities } \\
\text { (e.g., aged care or mental health) or role } \\
\text { emerging site (Search 2) \# }\end{array}$ \\
\hline 5. Geographical location & $\begin{array}{l}\text { Facilities in countries with similar } \\
\text { healthcare contexts, e.g., Australia, } \\
\text { Canada, United Kingdom, United States } \\
\text { and some European countries }\end{array}$ & All other countries \\
\hline 6. Publication date & $\begin{array}{l}\text { Published from 1990-present } \\
\text { (23 August 2017) }\end{array}$ & Studies published before 1990 \\
\hline 7. Duplication of data & & $\begin{array}{l}\text { - Data duplicated in another paper \# } \\
\text { - Systematic review paper not offering } \\
\text { any new data analysis \# }\end{array}$ \\
\hline
\end{tabular}

Note:

\# Criteria in italics added during final selection process for clarification (criteria 2 and 7) and expanded search (criteria 4)

\section{Study selection}

Records (title, full reference and abstract, where available) from database searches were extracted into EndNote X7 by the first author (EB). Two authors (EB and KS) then independently screened all records according to eligibility criteria (see Table 2) and full text papers were retrieved where either EB or KS (or both) rated them as "maybe" or "yes" for inclusion. Two authors (EB and KS) rated the full text of all remaining papers independently, using the same criteria. Results were compared and any disagreements resolved via discussion, resulting in minor clarifications to exclusions in criteria 2. Criteria 7 was also added at this time for increased clarity (shown in italics in Table 2). Papers were included if they were published in any language, however no selected papers required translation into English. On review of selected papers, it was determined that criterion 4 was unnecessarily restrictive, as many placement locations were not specified in published papers. EB re-screened all original records using an 
agreed revised criterion 4 (Search 2). EB and KS then rated additional full papers for eligibility using the same methods described above.

\section{Data extraction and quality assessment}

Two authors (EB \& KS) jointly completed data extraction and quality ratings for one study and refined any definitions and uncertainty of rating. Full data extraction was completed by EB — see Table 3 for a summary of data extracted. Concurrently, the risk of bias was assessed using the McMaster Guidelines for Critical Review Form: Quantitative Studies (Law et al., 1998), as it has been utilized in other papers regarding student supervision topics (e.g., Briffa \& Porter, 2013; Loewen et al., 2017). Interrater reliability of this protocol has been reported as $75-86 \%$ (Law et al., 2014). The adaptation of the scale by Lekkas et al. (2007), which rates studies on 14 items, scoring 1 for yes, meets criteria, or 0 for no, unmet criteria, was used. KS then independently completed the same process of data extraction and qualitative assessment for five (22\%) papers in accordance with the guidelines of Schlosser, Wendt and Sigafoos (2007). Inter-rater agreement for quality ratings of papers was on average $90 \%$, with a range per paper of $11-14 / 14$ items agreed.

Table 3

Summary of Data Extracted ftrom Included Articles

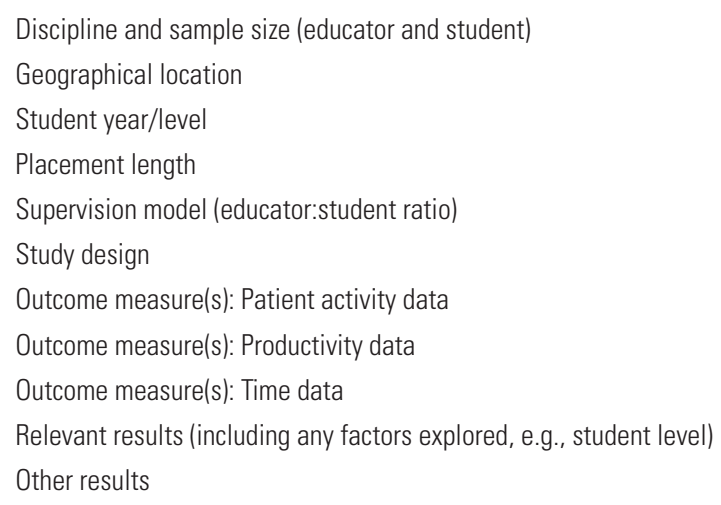

\section{Analysis}

Studies were included in further analysis of one or more outcome measures if they reported sample size, a mean calculation from the student and non-student period and either standard deviation, $p$ value or $t$ value; or if these could be calculated from raw data or other variance indicators (e.g., 95\% confidence interval for mean) using Microsoft Excel 2010. One author was contacted but was unable to provide the additional descriptive statistics required. In accordance with Borenstein, Hedges, 
Higgins and Rothstein (2009), studies including multiple student placement periods (but only one non-student period) were combined, and this synthesized mean and, if available, standard deviation, used in analysis. A similar process was used where multiple non-student periods were reported with a single student placement period. Given the diversity of data measurement reporting (e.g., activity by worked hour or per day), the effect size of student versus non-student periods was calculated using standardized mean difference and 95\% confidence interval (CI) using the Hedges' $g$ adjustment for small sample size (Borenstein et al., 2009).

\section{Synthesis of results and additional analyses}

In accordance with Liberati et al. (2009), to increase statistical power and strength of results, meta-analysis of data for activity, time and/or productivity was planned if more than two studies utilised similar outcome measures within an area of measurement. Sufficient data was found to be available for patient activity levels, direct clinical time, clinical billed units and direct time per patient. Comprehensive Meta-Analysis Software (CMA) Version 3.0 (Borenstein, Hedges, Higgins, \& Rothstein, 2014) was the software selected for meta-analysis. A random effects model was used for all meta-analyses, as studies differed explicitly (e.g., supervision model) and possibly implicitly (e.g., supervisor experience level, which was seldom reported), and it was considered highly unlikely all studies shared the same true effect size (Borenstein et al., 2009). Correlation between student placement and non-student period data was only reported in Ozelie, Janow, Kreutz, Mulry and Penkala (2015), who found a correlation of 0.75. Given that this measure of the statistical relationship between data was required to synthesise a number of studies, the robustness of using this figure was tested using sensitivity analysis, whereby various correlation coefficient values $(0.00,0.25,0.50,0.75$ and 0.99$)$ were imputed, and associated results examined. For each meta-analysis, the $\mathrm{I}^{2}$ measure of consistency, reported as a percentage from zero to 100 , was reviewed, providing some indication of study difference due to heterogeneity as opposed to chance (Borenstein, Higgins, Hedges, \& Rothstein, 2017). Funnel plots were visually inspected to estimate levels of publication bias but are not reported in analyses with less than 10 studies due to unreliability (Sterne et al., 2011). Pooled effects were computed where study methodologies were not deemed to vary substantially from each other. Sub-grouping by factors such as demographics (e.g., profession) was conducted post hoc if $\mathrm{I}^{2}$ was greater than $10 \%$ or there was wide variation in data and further analysis using these subgroups completed. An alpha level of 0.05 was used for all results. Moderator analysis of subgroups was not conducted due to the small number of studies in each subcategory. Three authors (EB, LM, KS) reviewed summary extracted data and discussed interpretation of results within the context of the qualitative ratings of papers. 


\section{Results}

\section{Study identification and selection}

Database searching identified 3,801 references, and seven were identified through other sources. Following removal of duplicates and assessment against eligibility criteria, 23 studies were selected for inclusion (see Figure 1 for details using the Preferred Reporting Items for Systematic Reviews and Meta-Analyses (PRISMA) flow diagram (Moher et al., 2009).

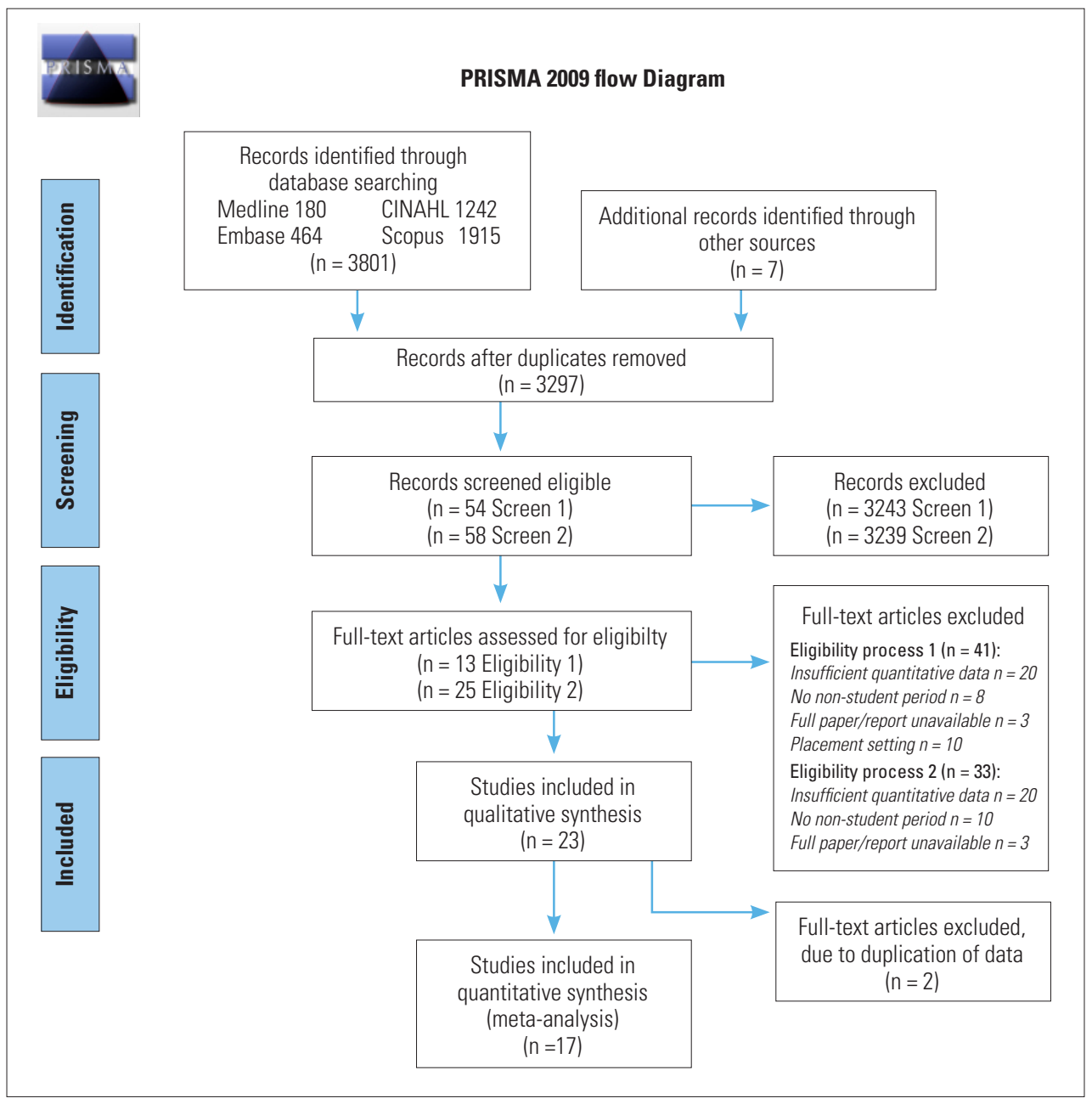

Figure 1. Process for selecting literature. 


\section{Characteristics of included studies}

\section{Population}

Table 4 summarises selected publications $(n=23)$ reporting the impacts of student supervision in nutrition and dietetics $(\mathrm{N} \& \mathrm{D}, \mathrm{n}=1)$, occupational therapy (OT, $\mathrm{n}=$ 4), physiotherapy (PT, $n=16)$ and speech pathology $(S P, n=3)$. Nine papers reported on placements completed in the United States, eight in Canada, four in Australia and one in the United Kingdom. One paper reported on data both from Canada and the United States. Sample size ranged from 2 to 114 clinicians/datasets (see Table 4), with a median of 18 from 22 papers (one paper did not specify). Seven of the eight largest studies $(\mathrm{n}>30)$ reported on PT placements.

\section{Intervention (exposure)}

Studies investigated placements utilising various student:placement supervisor ratios. The traditional one-to-one model of supervision was most commonly featured (see Table 4), with seven papers using this exclusively, five papers (Bristow \& Hagler, 1994; Graham, 1991; MacDonald, Cox, \& Bartlett, 2002; Pivko, Abbruzzese, Duttaroy, Hansen, \& Ryans, 2016; Rodger, Stephens, Clark, Ash, \& Graves, 2011) using student and clinician numbers or descriptions consistent with a one-to-one or one-to-two ratio and a further four comparing one-to-one with two-to-one supervision. Four studies only included clinicians supervising multiple students. There was no observable pattern of the supervision model used in relation to the profession studied, student year level (see Table 4) or the geographical location of the placements.

\section{Outcome}

There was variation in number and type of outcome measures reported (see Table 4) as well as the associated definitions of activities. Of the studies selected in the literature search, 18 had sufficient information to be considered for at least one meta-analysis (shown as italicised outcome measures in Table 4. See Tables 5 to 9 for specific data details). For some papers, data was published separately according to sub-categories (e.g., student level), so these were utilised separately in meta-analysis if adequate statistics were available. In some domains, there was insufficient data available to complete any further analysis.

\section{Data collection approach}

Twelve studies collected data prospectively, nine reported retrospective analyses of routinely collected data and the remaining two could not be determined. Study collection periods varied from 10 days to 4 years (see Table 4), with more than half the studies (17/23) studying the whole placement period (from 3 weeks to 12 months in length). The majority (18/23) of non-student collection periods were equivalent in length to the matched student placement. 


\section{Quality assessment}

Quality scores are shown in Table 4. Scores ranged from 4-11 out of 14, with a median of 9 . Some notable strengths across most included papers were clear study purpose(s) and reported educational importance. Justification of sample size and detailed description of intervention (in this paper interpreted as student supervision model and teaching practices) were mostly absent from included studies. Overall, the quality of research was fairly low, and a high risk of bias should be assumed. However, it was determined that computing effect sizes from papers scoring at least seven ( $\mathrm{n}=17$ papers) would facilitate study analysis and comparison in a more robust way than other methods (Valentine, Pigott, \& Rothstein, 2010), as well as potentially raise the level of evidence available, particularly if data could be pooled in meta-analysis. 
IMPACT OF PLACEMENTS ON ALLIED HEALTH TIME USE AND PRODUCTIVITY

\begin{tabular}{|c|c|c|c|c|c|c|}
\hline ә.0วs אᄉำ| & 으 & 웅 & & $=$ & os & 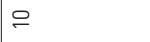 \\
\hline 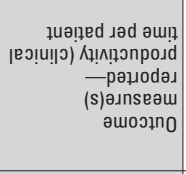 & 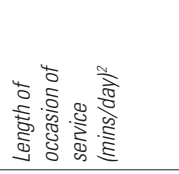 & $\frac{⿱}{2}$ & 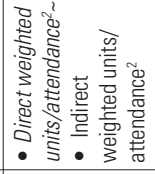 & $\frac{m}{2}$ & $\frac{\infty}{2}$ & 愛 \\
\hline 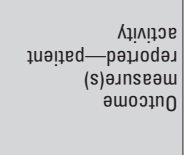 & 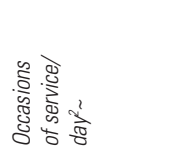 & $\frac{m}{2}$ & 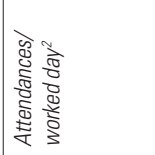 & 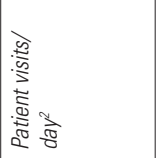 & 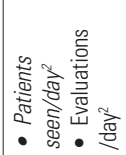 & 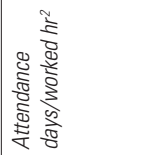 \\
\hline 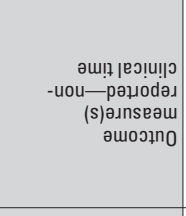 & 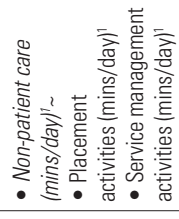 & 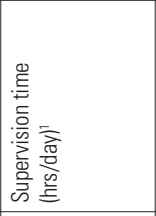 & 爱 & $\frac{n}{z}$ & $\frac{\infty}{z}$ & 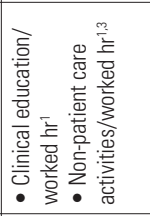 \\
\hline 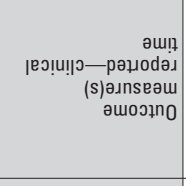 & 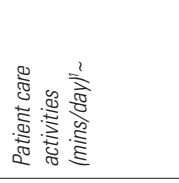 & 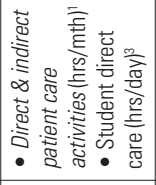 & & $\frac{\Upsilon}{z}$ & 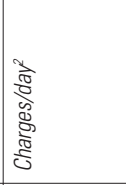 & 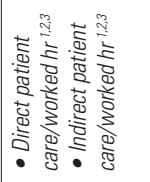 \\
\hline 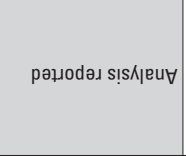 & 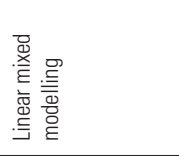 & 辛 & 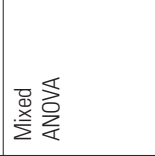 & 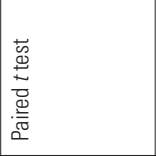 & 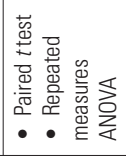 & 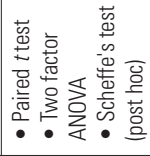 \\
\hline 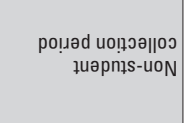 & 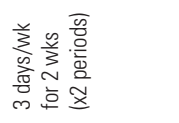 & 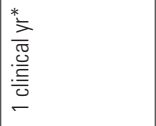 & 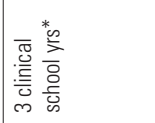 & $\underline{\underline{\underline{E}}}$ & $\underset{⿱ 亠 䒑}{3}$ & 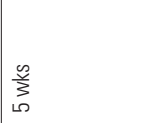 \\
\hline 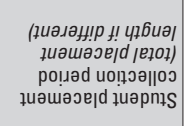 & 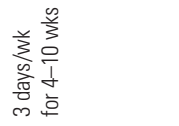 & 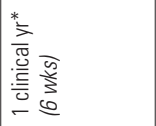 & 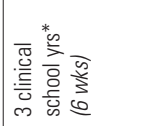 & $\begin{array}{l}\infty \\
3 \\
o p \\
q\end{array}$ & $\underset{⿱ 亠 䒑}{3}$ & 竞 \\
\hline \# |әрош uo!s!̣ıәdns & $\frac{⿱ 亠 ⿰ 口 口}{2}$ & $\frac{⿱}{2}$ & $\mp \stackrel{N}{\infty}$ & $\check{I}$ & $\stackrel{\infty}{\check{C}} \bar{i}$ & 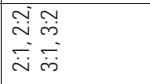 \\
\hline деәК ұиәрпн & 焉 & ले & ले & 高 & 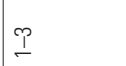 & $\stackrel{I}{\sim}$ \\
\hline 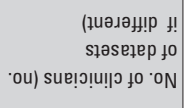 & $\mathscr{N} \stackrel{\overline{\frac{m}{t}}}{=}$ & 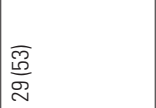 & 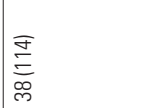 & $\stackrel{\infty}{\infty}$ & مـ & 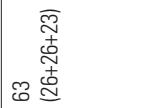 \\
\hline uo!ssejold & $\frac{\not}{2}$ & ta & 5 & a & 5 & 5 \\
\hline sıoчın & 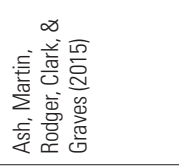 & 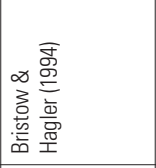 & 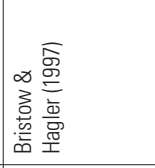 & 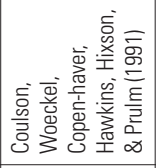 & 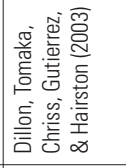 & 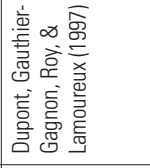 \\
\hline 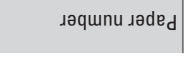 & - & N & $m$ & ब & in & $\infty$ \\
\hline
\end{tabular}


IMPACT OF PLACEMENTS ON ALLIED HEALTH TIME USE AND PRODUCTIVITY

\begin{tabular}{|c|c|c|c|c|c|c|}
\hline әرоэs אᄉ & $\infty$ & $\sigma$ & $\infty$ & n & $\infty$ & 0 \\
\hline 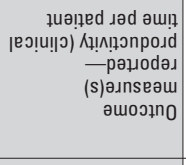 & $\frac{c}{2}$ & $\frac{⿱}{z}$ & 愛 & 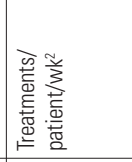 & 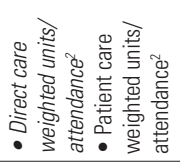 & $\frac{9}{2}$ \\
\hline 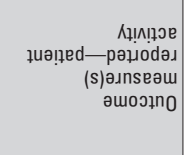 & 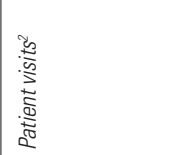 & $\frac{m}{z}$ & $\frac{m}{z}$ & 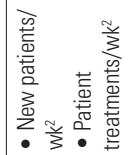 & 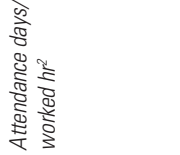 & $\frac{n}{z}$ \\
\hline 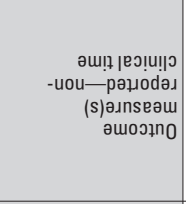 & & 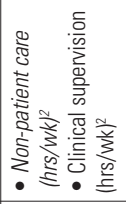 & 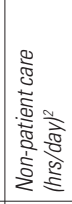 & $\frac{a}{z}$ & 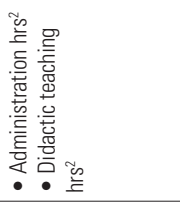 & 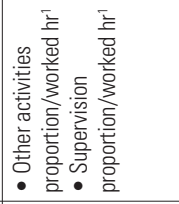 \\
\hline 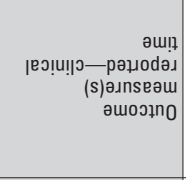 & 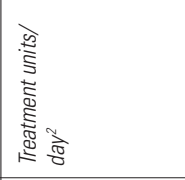 & 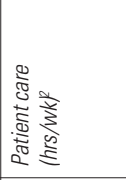 & 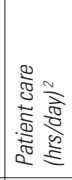 & 兴 & 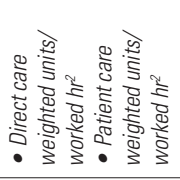 & 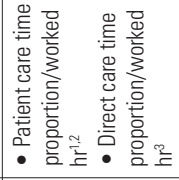 \\
\hline 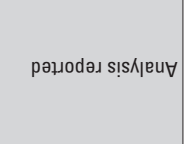 & 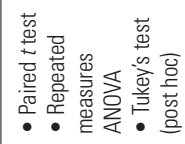 & 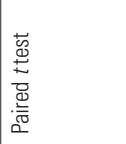 & 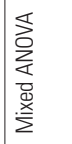 & 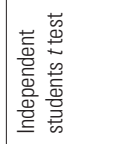 & $\frac{m}{z}$ & 愛 \\
\hline 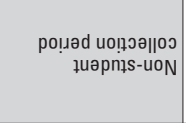 & $\begin{array}{l}\infty \\
\frac{0}{2} \\
50 \\
0 \\
0 \\
3 \\
2\end{array}$ & 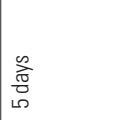 & $\underset{\substack{* \\
\infty \\
⿱ 亠 乂}}{*}$ & 竞 & $\stackrel{\text { 美 }}{-}$ & $\stackrel{\text { s. }}{3}$ \\
\hline 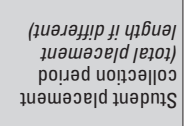 & 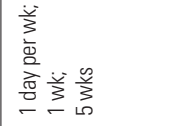 & 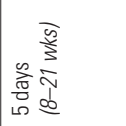 & 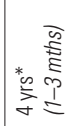 & 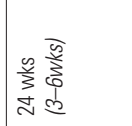 & 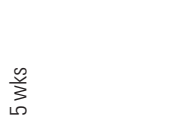 & 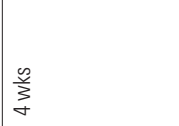 \\
\hline \# |әрош uo!s!̣ıәdns & $\frac{q}{2}$ & $\check{-}$ & $\check{-}$ & 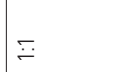 & $\bar{i}$ & $\stackrel{\infty}{\check{\check{\sim}}}$ \\
\hline деәК ұиәрпาร & 言兽 & i & 垔 & $\stackrel{m}{\sim}$ & $m$ & $\theta$ \\
\hline 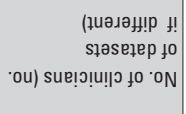 & $\begin{array}{l}\text { क्o } \\
0 \\
+ \\
\stackrel{0}{0} \\
\infty\end{array}$ & $=$ & $=$ & $\stackrel{\text { d }}{=}$ & $\infty$ & $\bar{m}$ \\
\hline uo!ssejold & 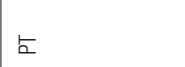 & कि & is & 는 & ㄴ. & เ \\
\hline sıoung & 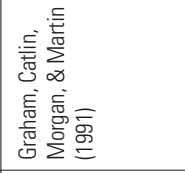 & 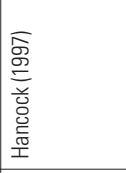 & 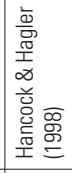 & 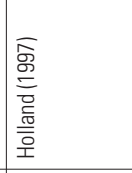 & 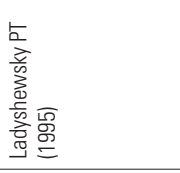 & 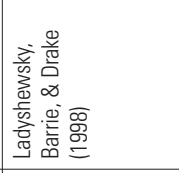 \\
\hline „əqunu „əde $d$ & $r$ & $\infty$ & 0 & $\div$ & $=$ & $\simeq$ \\
\hline
\end{tabular}


IMPACT OF PLACEMENTS ON ALLIED HEALTH TIME USE AND PRODUCTIVITY

\begin{tabular}{|c|c|c|c|c|c|c|c|}
\hline כ.0эs & 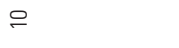 & or & 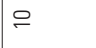 & ما & 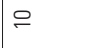 & 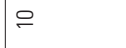 & or \\
\hline 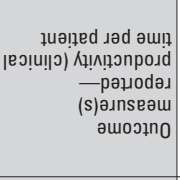 & 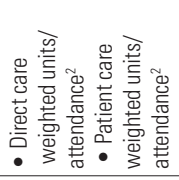 & 無 & 爱 & 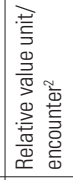 & 無 & 無 & 愛 \\
\hline 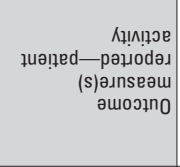 & 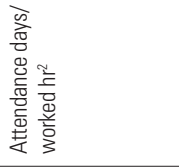 & 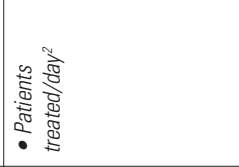 & \begin{tabular}{|l}
$\frac{1}{5}$ \\
$\frac{2}{2}$ \\
$\frac{2}{2}$ \\
$\frac{5}{5}$ \\
\end{tabular} & 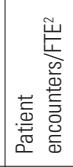 & $\stackrel{\Upsilon}{z}$ & 愛 & $\frac{m}{z}$ \\
\hline 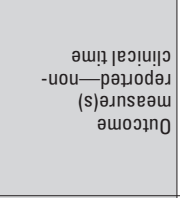 & & 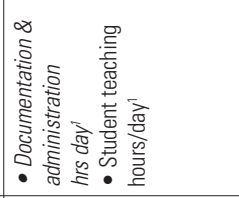 & 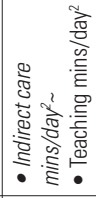 & 無 & 無 & $\frac{\Upsilon}{2}$ & 無 \\
\hline 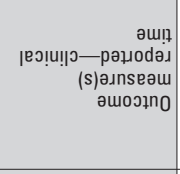 & 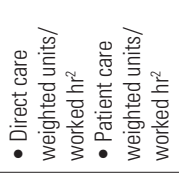 & 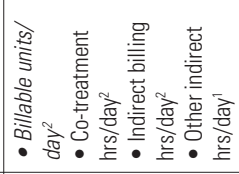 & 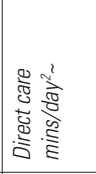 & 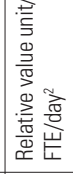 & 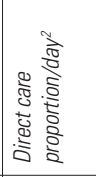 & 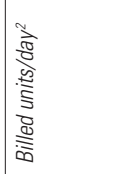 & 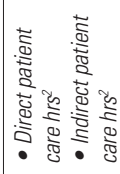 \\
\hline 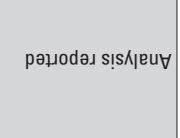 & 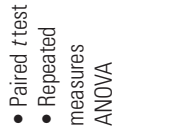 & 無 & 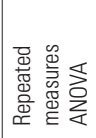 & 愛 & 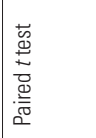 & 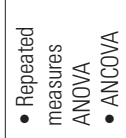 & 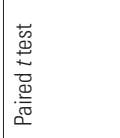 \\
\hline 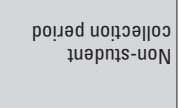 & 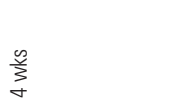 & 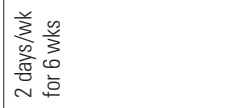 & 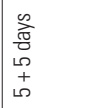 & 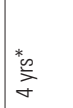 & 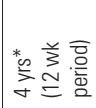 & ${ }_{\substack{* \\
\sum_{m}^{*}}}$ & $\begin{array}{l}\text { 悉 } \\
\sum_{m}^{\infty}\end{array}$ \\
\hline 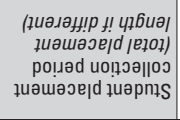 & $\stackrel{\substack{3 \\
\hdashline}}{4}$ & 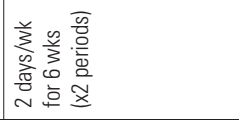 & 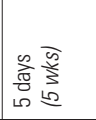 & 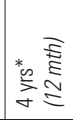 & 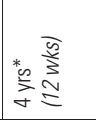 & 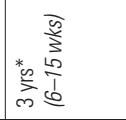 & 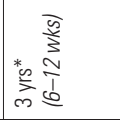 \\
\hline \# |әрош uo!ș!Mədns & $\frac{m}{z}$ & $\stackrel{\infty}{\check{i}} \bar{i}$ & $\frac{9}{z}$ & $\frac{m}{z}$ & $\check{\check{-}}$ & $\stackrel{\infty}{\check{C}}$ & $\frac{q}{2}$ \\
\hline деәК ұиәрпн & छे & 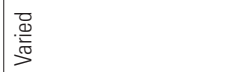 & $\sim$ & $\begin{array}{l}\stackrel{E}{\underline{\underline{E}}} \\
\underline{\underline{E}}\end{array}$ & $\frac{N}{\bar{w}}$ & 1 & 焉里 \\
\hline 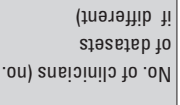 & 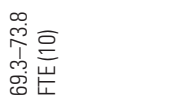 & $m$ & 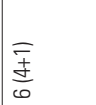 & 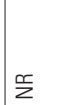 & 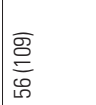 & ని & $\approx$ \\
\hline uo!ssajold & L & 5 & t & 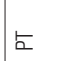 & t5 & 5 & 5 \\
\hline sıouाn & 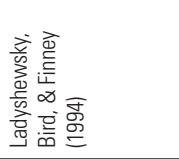 & 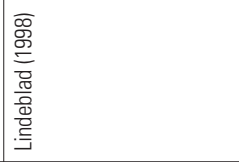 & 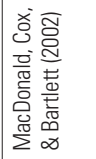 & 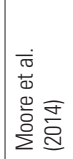 & 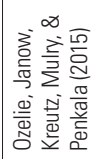 & 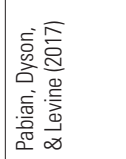 & 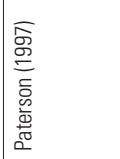 \\
\hline 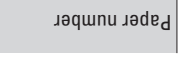 & $\stackrel{m}{2}$ & \pm & $\stackrel{2}{\llcorner}$ & 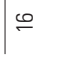 & $=$ & 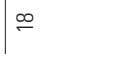 & $\stackrel{\rho}{\circ}$ \\
\hline
\end{tabular}


IMPACT OF PLACEMENTS ON ALLIED HEALTH TIME USE AND PRODUCTIVITY

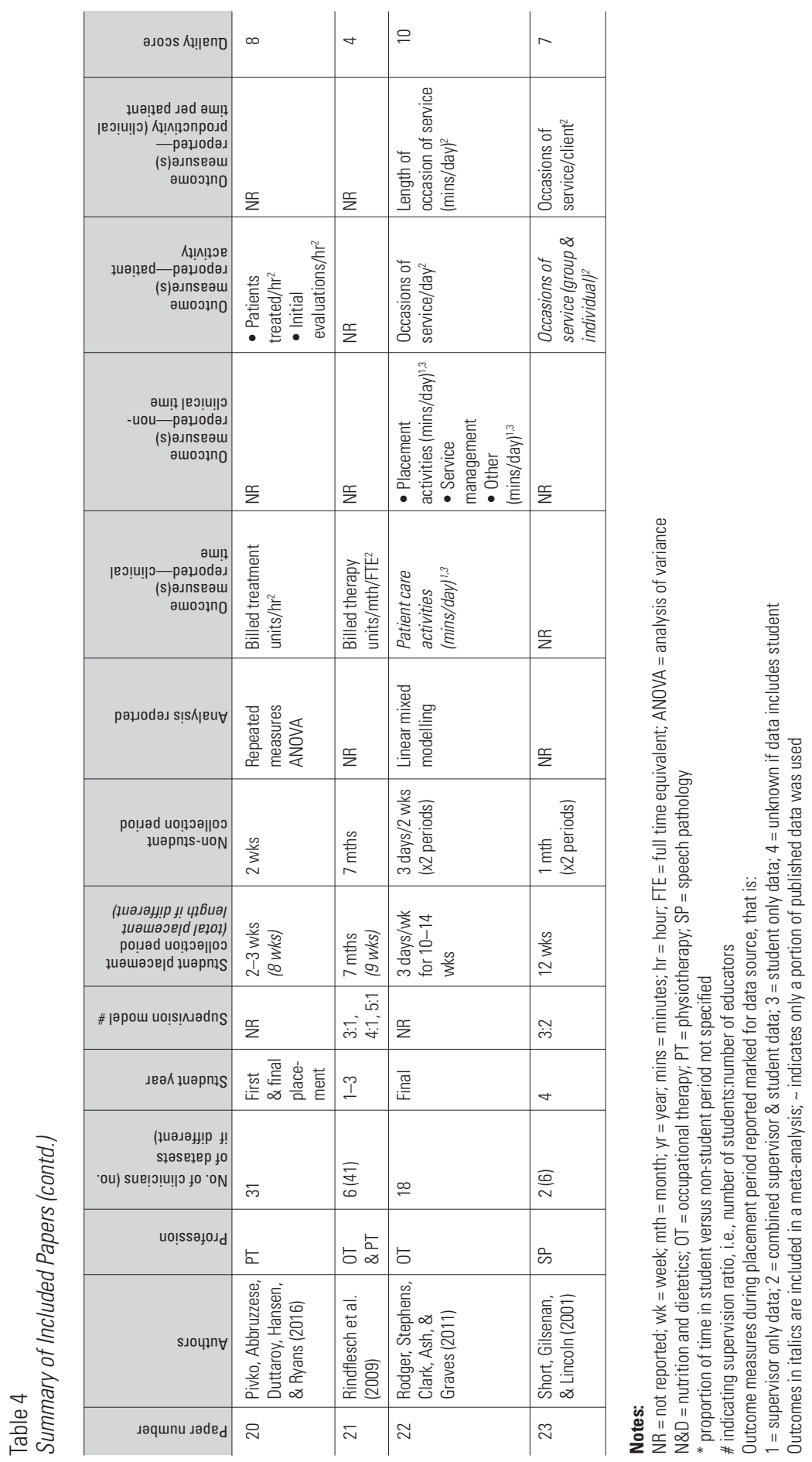


IMPACT OF PLACEMENTS ON ALLIED HEALTH TIME USE AND PRODUCTIVITY

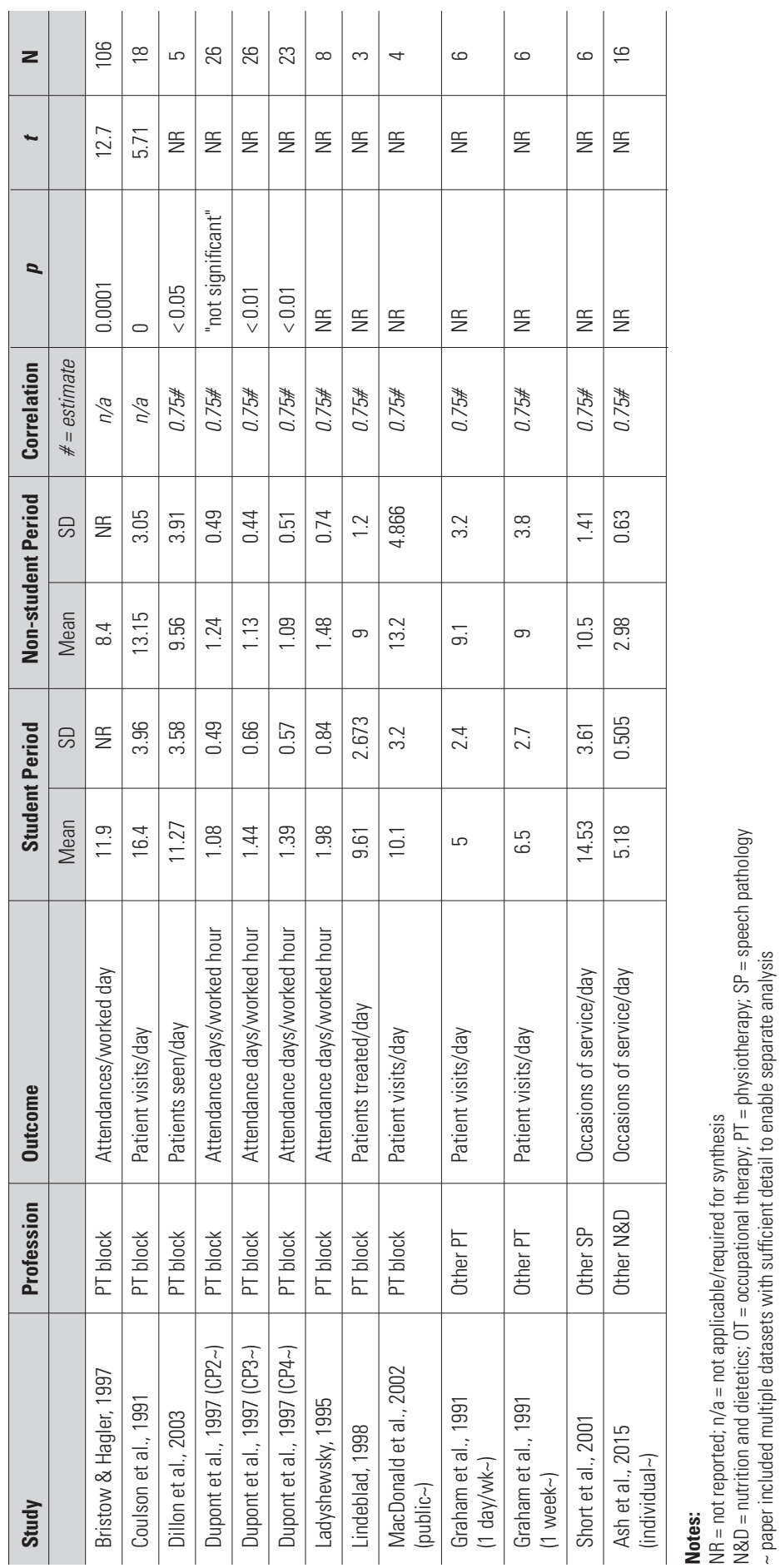


IMPACT OF PLACEMENTS ON ALLIED HEALTH TIME USE AND PRODUCTIVITY

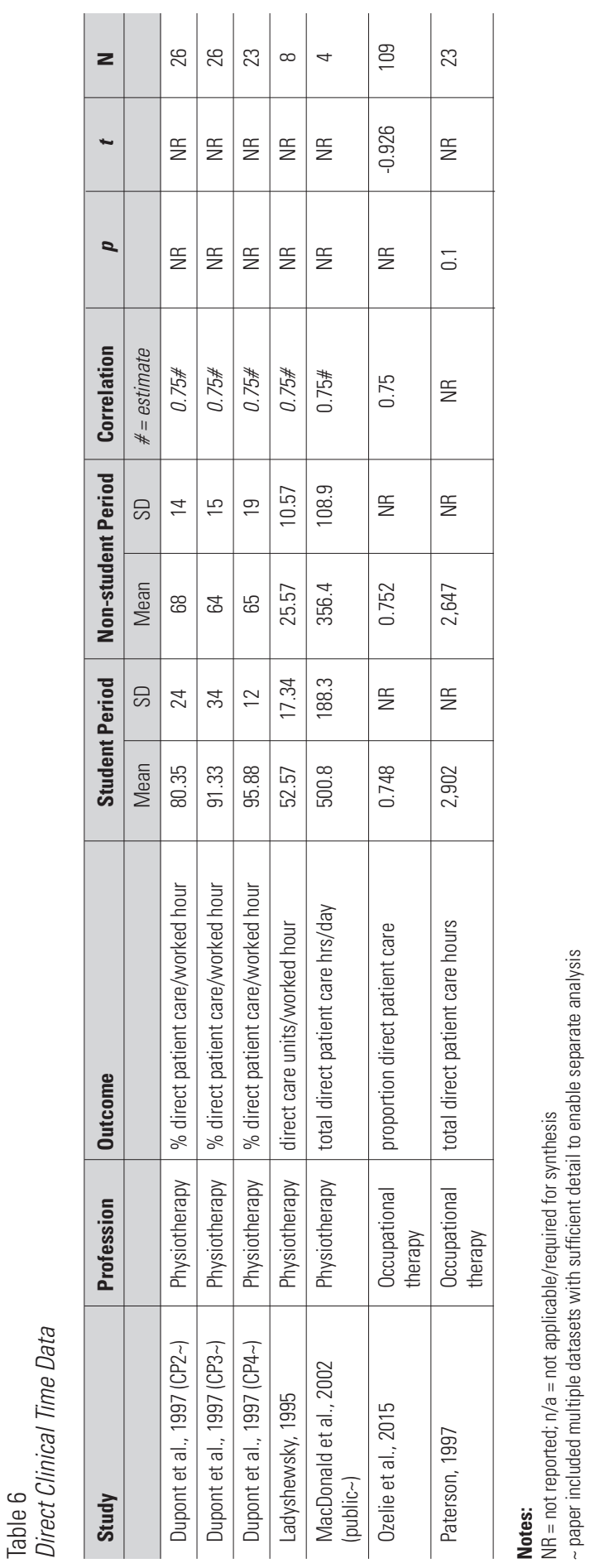


IMPACT OF PLACEMENTS ON ALLIED HEALTH TIME USE AND PRODUCTIVITY

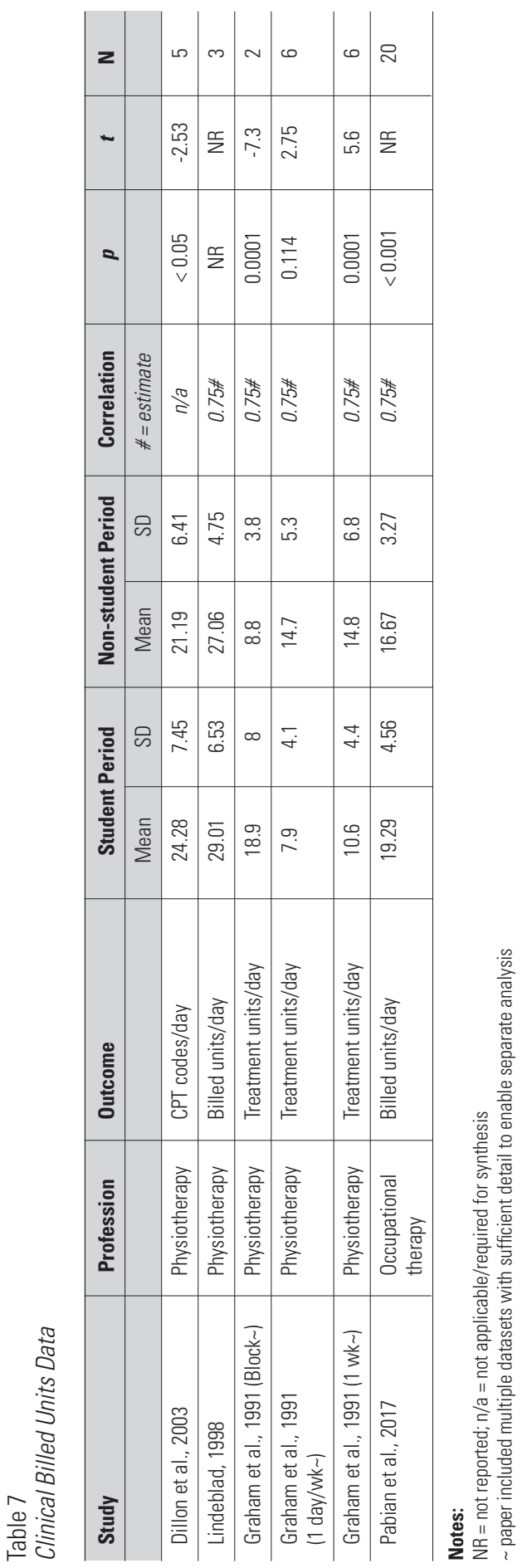




\section{IMPACT OF PLACEMENTS ON ALLIED HEALTH TIME USE AND PRODUCTIVITY}

Table 8

Teaching Time Data

\begin{tabular}{|c|c|c|c|c|c|c|}
\hline \multirow[t]{2}{*}{ Study } & \multirow[t]{2}{*}{ Profession } & \multirow[t]{2}{*}{ Outcome } & \multicolumn{2}{|c|}{ Student Period } & \multirow[t]{2}{*}{$\mathbf{N}$} & \multirow[t]{2}{*}{ Mins/day } \\
\hline & & & Mean & SD & & \\
\hline Ash et al., 2015 & $\begin{array}{l}\text { Nutrition \& } \\
\text { dietetics }\end{array}$ & $\begin{array}{l}\text { Placement activities } \\
\text { mins/day }\end{array}$ & 45.88 & 11.31 & 18 & 45.9 \\
\hline Bristow \& Hagler, 1994 & Physiotherapy & Supervision hrs/day & 1.0 & & 30 & 60.0 \\
\hline $\begin{array}{l}\text { Bristow \& Hagler, } 1994 \\
\text { (SCl only\#) }\end{array}$ & Physiotherapy & Supervision hrs/day & 2.0 & & $N R$ & 120.0 \\
\hline $\begin{array}{l}\text { Bristow \& Hagler, } 1994 \\
\text { (Neuro only\#) }\end{array}$ & Physiotherapy & Supervision hrs/day & 0.7 & & $N R$ & 39.6 \\
\hline $\begin{array}{l}\text { Bristow \& Hagler, } 1994 \\
\text { (MVC only\#) }\end{array}$ & Physiotherapy & Supervision hrs/day & 0.8 & & 6 & 48.0 \\
\hline $\begin{array}{l}\text { Bristow \& Hagler, } 1994 \\
\text { (B/P only\#) }\end{array}$ & Physiotherapy & Supervision hrs/day & 0.76 & & 13 & 45.6 \\
\hline Dupont, 1997 (CP2\#) & Physiotherapy & \%Clin Ed/worked hour & 62 & & 26 & 297.6 \\
\hline Dupont, 1997 (СР3\#) & Physiotherapy & \%Clin Ed/worked hour & 59 & & 26 & 283.2 \\
\hline Dupont, 1997 (CP4\#) & Physiotherapy & \%Clin Ed/worked hour & 60 & & 23 & 288.0 \\
\hline Hancock, 1997 & Speech pathology & $\begin{array}{l}\text { Clinical supervision hours/ } \\
\text { week }\end{array}$ & 9.27 & 4.99 & 11 & 111.2 \\
\hline Ladyshewsky, 1995 & Physiotherapy & didactic teaching $/ 5$ wks & 27.58 & & 8 & 55.2 \\
\hline Ladyshewsky et al., 1998 & Physiotherapy & $\%$ supervision/worked hour & 0.57 & & 31 & 273.6 \\
\hline Lindeblad, 1998 & Physiotherapy & student teaching hours/day & 0.417 & 0.33 & 3 & 25.0 \\
\hline Macdonald et al., 2002 & Physiotherapy & teaching/day & 235.3 & 211.8 & 4 & 235.3 \\
\hline Rodger et al., 2011 & $\begin{array}{l}\text { Occupational } \\
\text { therapy }\end{array}$ & $\begin{array}{l}\text { placement activities } \\
\text { mins/day }\end{array}$ & 49.82 & & 18 & 49.8 \\
\hline
\end{tabular}

\section{Notes:}

$\mathrm{NR}=$ not reported

\# paper included multiple datasets with sufficient detail to enable separate analysis 
IMPACT OF PLACEMENTS ON ALLIED HEALTH TIME USE AND PRODUCTIVITY

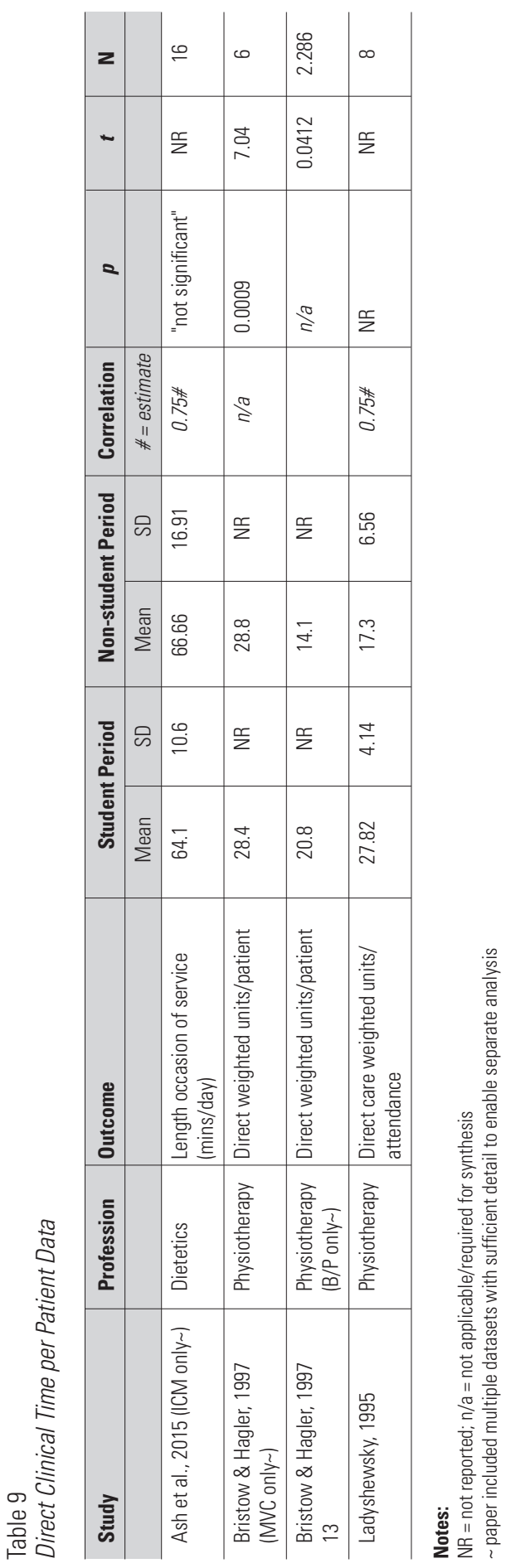




\section{Results of data analysis}

Quantitative results are presented below in relation to our objectives-A: patient activity levels, B: clinician time usage and C: productivity — linking time and activity data.

\section{Objective A: Patient activity levels}

Effect sizes (ES) for papers reporting activity levels during student placement versus nonstudent periods varied as to whether they favoured the student or non-student period, or showed no difference (range -1.153 to 3.533, see Figure 2). Individual ES were significantly higher in student periods in seven datasets and significantly lower in three datasets (total 13 datasets from 10 papers). Meta-analysis revealed a significant pooled ES of $0.456(95 \% \mathrm{CI}=0.021-0.891, p=0.04)$. There was evidence of heterogeneity between studies $\left(\mathrm{I}^{2}=46.2 \%\right)$. A funnel plot for this data (see Figure 3 ) did not indicate systematic publication bias between the studies.

Post hoc sub-analysis: Given the noted heterogeneity, subgroups were developed post hoc using profession and placement length: 1) nutrition and dietetics (one dataset), 2) physiotherapy short placements (two datasets, total sample $n=12, I^{2}=0 \%$ ), 3) speech pathology (one dataset) and 4) physiotherapy block placements (nine datasets, total sample $\mathrm{n}=229, \mathrm{I}^{2}=0 \%$ ). Pooled ES for subgroup 2, PT short placements, significantly favoured the non-student period (ES $-0.836,95 \% \mathrm{CI}=-1.382$ to $-0.290, p=0.003$ ). Pooled ES for subgroup 4, PT 4- to 8-week blocks, significantly favoured student placement periods (ES 0.418, 95\% CI $=0.011-0.826, p=0.044$ ). The weighted mean increase in activity levels was $24.1 \%$. Within this subgroup, two results with a negative ES sampled second-year student placements, whereas all remaining studies, except Dillon, Tomaka, Chriss, Gutierrez and Hairston (2003) and Lindeblad (1998) exclusively studied later-year students.

\section{Objective B: Time use}

ES for comparisons of direct clinical time data (combining supervisor and student input during student placement period) ranged from -0.088-1.651 (see Figure 4). Individual ES were significantly higher in student periods in five datasets and showed no difference in two datasets (total $=$ seven datasets from five papers). Meta-analysis revealed a significant pooled ES of $0.703(95 \% \mathrm{CI}=0.241-1.164, p=0.003)$. There was no evidence of heterogeneity between studies $\left(\mathrm{I}^{2}=0 \%\right)$.

Post hoc sub-analysis: Given data variation, subgroups were investigated post hoc using profession: 1) occupational therapy (OT, two datasets, total sample $\mathrm{n}=132, \mathrm{I}^{2}=0.0 \%$ ) and 2) physiotherapy block placements (PT, five datasets, total sample $n=87, I^{2}=7.6 \%$ ). Pooled ES for subgroup 1, OT, was not significantly different to zero (ES 0.042, 95\% $\mathrm{CI}=-0.292-0.377, p=0.804)$. Pooled ES for subgroup 2, PT, significantly favoured student placement periods (ES $0.962,95 \% \mathrm{CI}=0.524-1.400, p=0.000$ ). The weighted mean increase in direct clinical time for PT was $42.3 \%$.

Clinical billed units ES data from five papers ranged from -1.154 to 0.905 (see Figure 5). Individual ES were significantly higher in student periods in two datasets, two 
IMPACT OF PLACEMENTS ON ALLIED HEALTH TIME USE AND PRODUCTIVITY

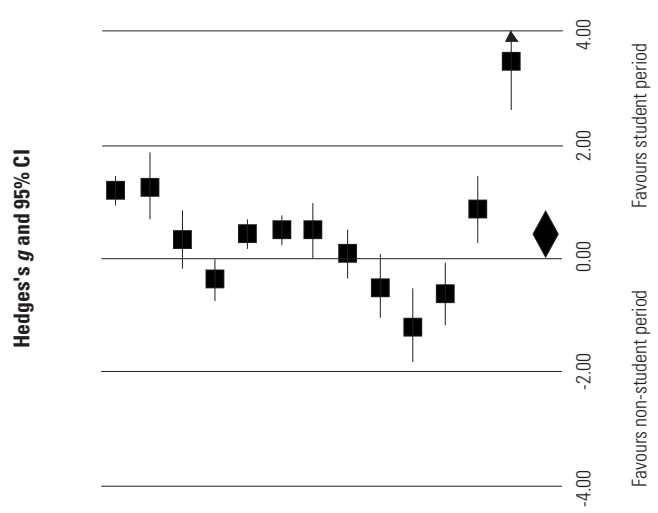

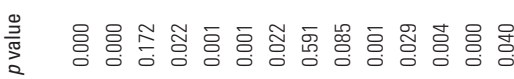

善

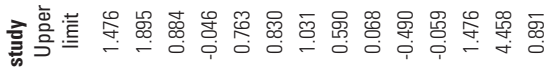
递

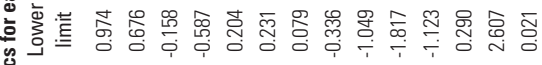
.

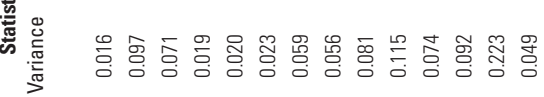

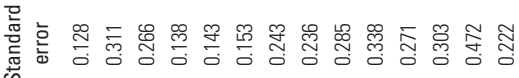

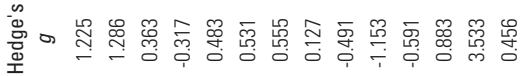

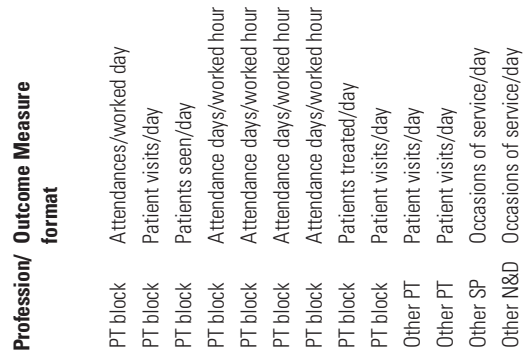




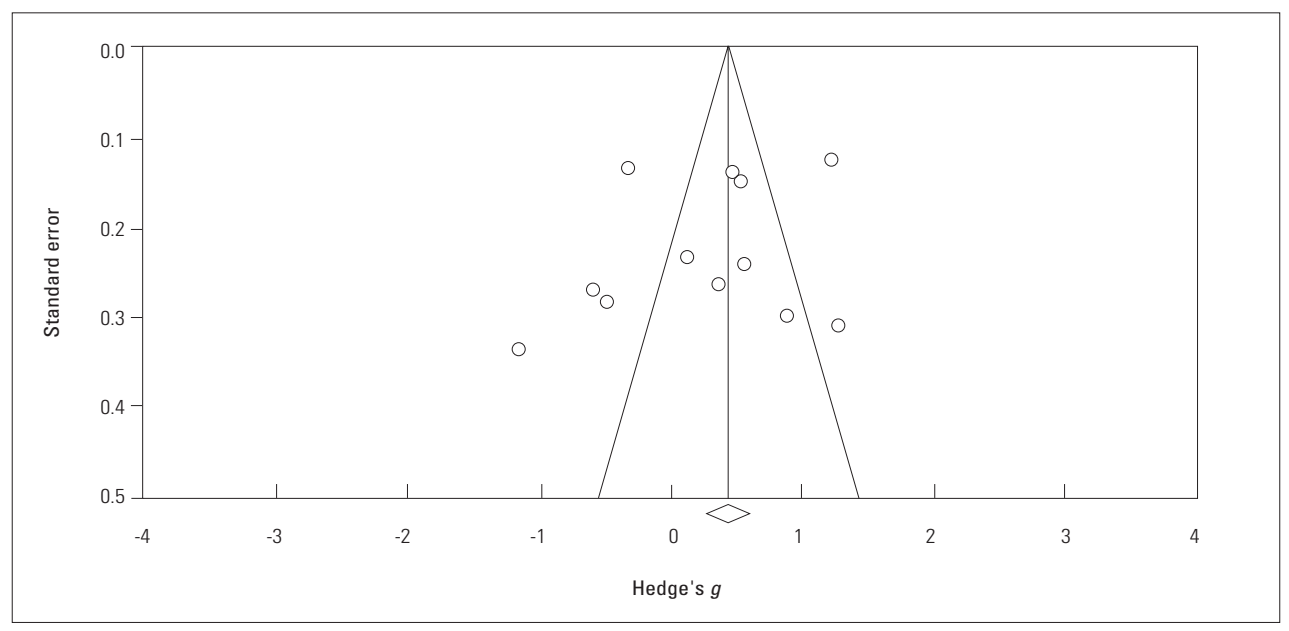

Figure 3. Patient activity levels funnel plot of standard error by Hedges's $g$.

significantly favoured the non-student period and one showed no difference. Metaanalysis revealed no significant difference in pooled results (ES $-0.014,95 \% \mathrm{CI}=$ $-0.673-0.645, p=0.966)$. There was evidence of heterogeneity between studies $\left(\mathrm{I}^{2}=\right.$ $15.9 \%)$. Further analysis was not conducted due to uncertainty regarding the similarity of included studies.

Eight papers reported the time supervisors specifically spent teaching students (e.g., giving feedback or demonstrating techniques). A meta-analysis could not be performed, however conversion of available data to time per day revealed that student teaching time ranged from 25 minutes to nearly 5 hours per day, with a median of 1 hour per day. No trends according to supervision model, profession, student level or block length were noted.

Insufficient data or papers were available to calculate comparable effect sizes in the categories of indirect clinical, combined direct and indirect clinical or non-clinical time use.

\section{Objective C: Linking time and activity data to establish productivity}

ES for the amount of direct clinical time used per patient ranged from - 0.151 to 2.42 (see Figure 6). Individual ES were significantly higher, favouring student periods, in the three papers reporting on 5- to 6-week blocks in PT $(p<0.05)$ and showed no significant difference in the N\&D study. Meta-analysis revealed no significant difference in pooled results (ES 0.924, 95\% CI $=-0.022-1.870, p=0.056$ ). There was evidence of heterogeneity between studies $\left(\mathrm{I}^{2}=16.3 \%\right)$. Further analysis was not conducted.

Sensitivity analysis: Meta-analyses conducted using specified correlation coefficients showed pooled ES and 95\% CI varied (see Table 10) but rarely changed overall results 
IMPACT OF PLACEMENTS ON ALLIED HEALTH TIME USE AND PRODUCTIVITY
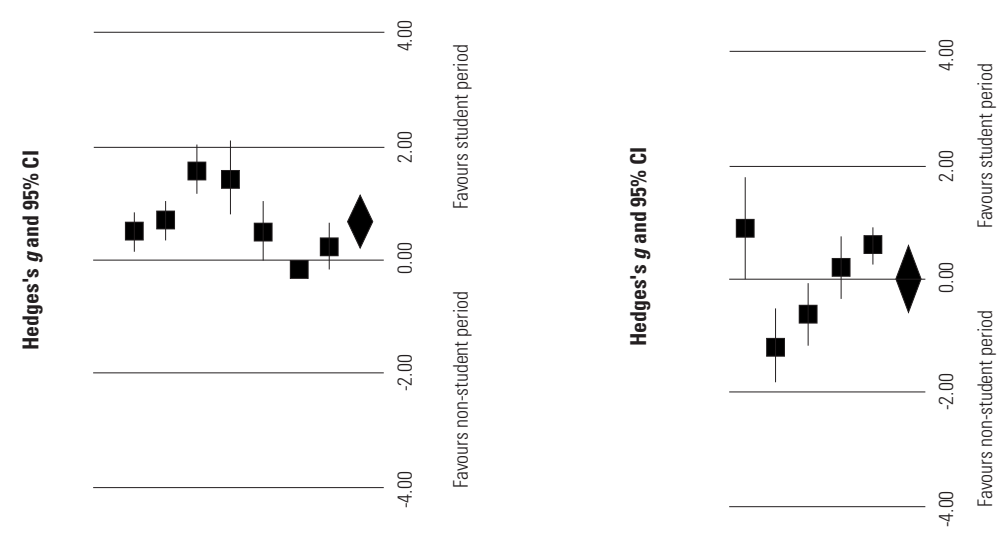

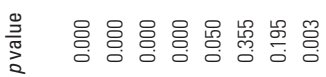

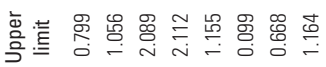

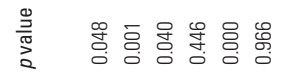

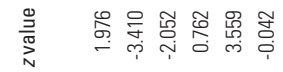

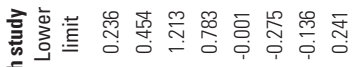

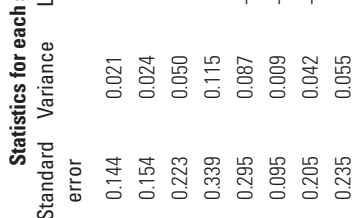

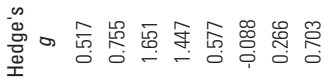

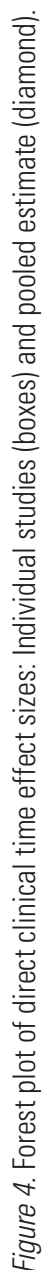

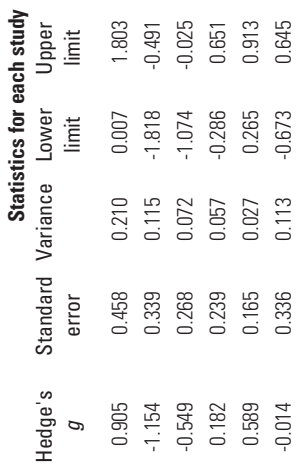
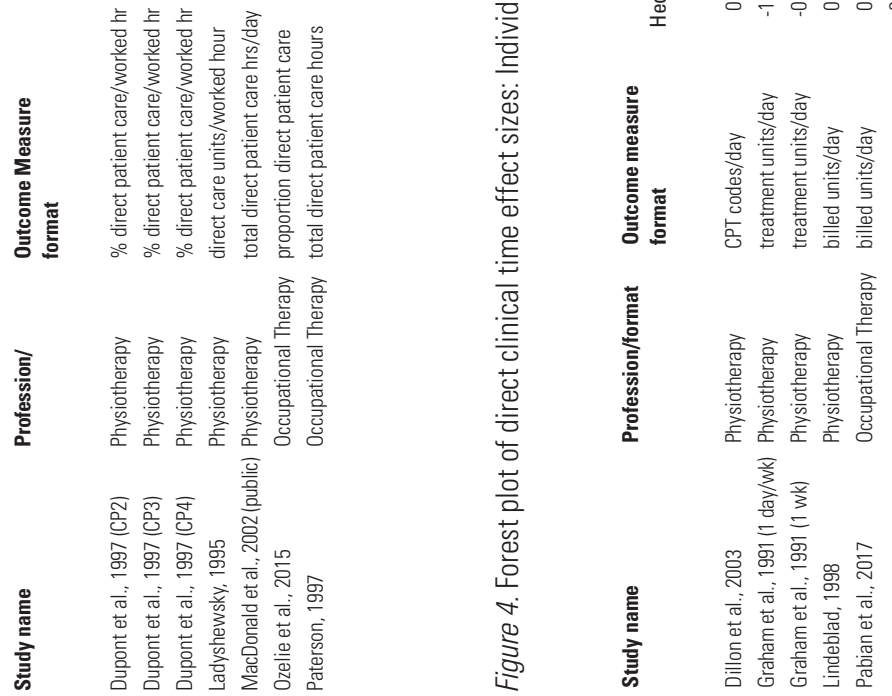

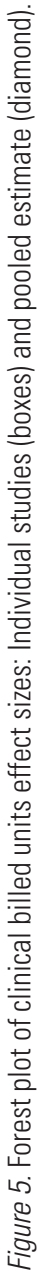


IMPACT OF PLACEMENTS ON ALLIED HEALTH TIME USE AND PRODUCTIVITY

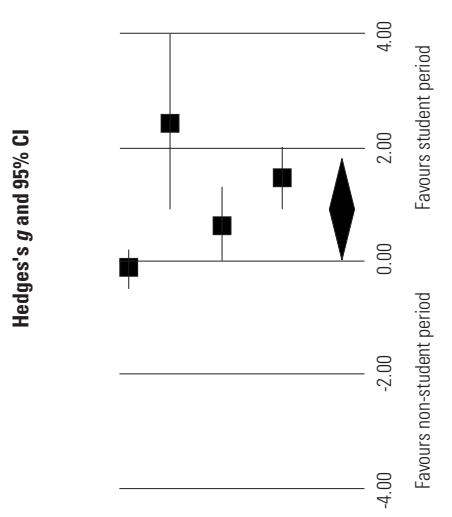

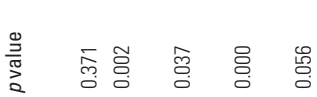

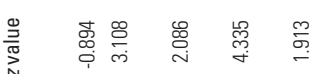

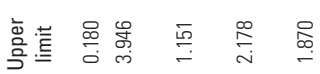

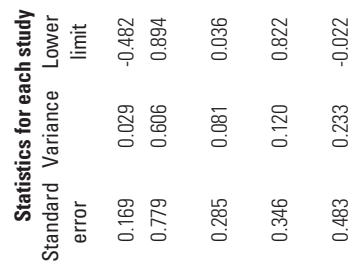

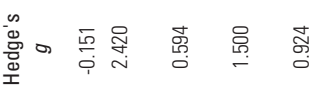

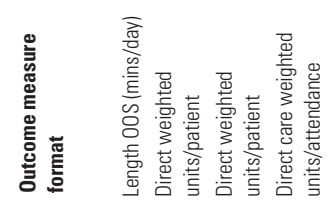

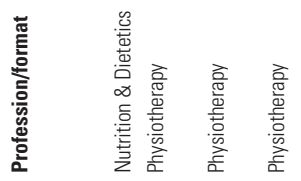

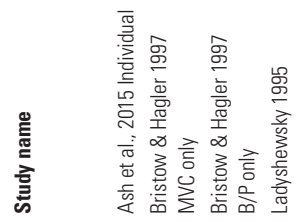

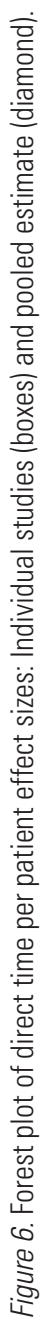

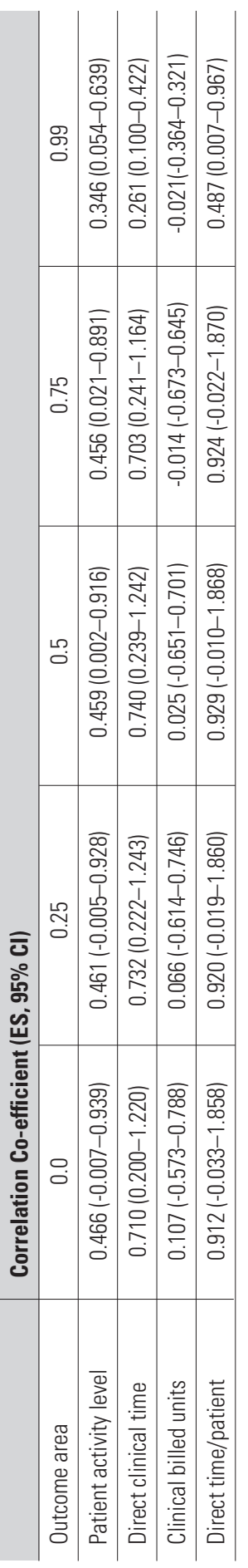


(e.g., ES consistently favoured the student period). Hence, the use of 0.75 , a known result from one paper, seemed the most suitable assumption for all calculations.

\section{Discussion}

This study has summarised data pertaining to understanding how supervising students affects patient activity levels, clinical time use and productivity of selected allied health clinicians. Meta-analysis has provided greater certainty in quantifying this impact across various measures used in healthcare services (see summary in Figure 7). Data from PT is most commonly reported and pooled, which is not surprising considering factors such as the workforce being larger than the other included professions (Pretorius, Karunaratne, \& Fehring, 2016) and PT possibly having a greater presence in public or private services where this data is highly valued. However, we have broadened the understanding of student impact in measures useful to service managers and clinicians in the other $\mathrm{AH}$ professions, which guides hypothesis generation for future research, more specifically, in relation to our research questions.

\begin{tabular}{l|l}
\hline \multirow{2}{*}{ Outcome } & Pooled results effect size\# (no. of datasets) \\
\hline Patient activity levels & $\uparrow$ during placements for whole group (13) \& PT blocks subgroup (9) \\
\cline { 2 - 2 } & $\downarrow$ during placements for PT short placements subgroup (2) \\
\hline \multirow{2}{*}{ iirect clinical time (with patient) } & $\uparrow$ during placements for whole group (7) \& PT blocks subgroup (5) \\
\cline { 2 - 2 } & No significant difference for OT subgroup (2) \\
\hline Clinical billed units & No significant difference (5) \\
\hline Productivity (direct time/patient) & No significant difference (4) \\
\hline
\end{tabular}

Note:

\# Difference between means of data collected during student placement periods versus non-student periods

Figure 7. Summary of effect size calculations.

\section{Objective A: Patient activity levels}

Meta-analysis indicated that a higher number of patients can be seen when allied health students are on placement. In the PT blocks subgroup, the difference for a clinician who normally sees 10 patients/day equates to an average additional two patients, that is, a total of over 12 patients/day being seen in conjunction with students. Whilst the exact contribution of students to this total is mostly unreported, variation in the contributing studies did seem to be partly explained by student level, with earlyyear students more commonly involved in a decline in patient activity levels, which is consistent with findings in Hall et al. (2015). Placement length may be another potentially limiting factor, with the two studies using shorter placements showing a negative impact on activity levels. When compared to the increases found in longer block placements, this provides some preliminary evidence for longer placements being preferred by employers, as in O'Brien et al. (2017). Further robust research in all 
disciplines is required to confirm the impact of students on patient activity levels, with careful consideration of variables such as student level and varying placement length to guide university curriculum design.

\section{Objective B: Clinician time usage}

The effect of students on clinician time use varied depending on the measure used (e.g., billed unit versus proportion of time per day) as well other factors such as the individual profession studied. Measures of indirect clinical and non-clinical time use (other than teaching) could not be further analysed.

Meta-analyses of direct clinical time in the selected professions showed that combined supervisor and student direct clinical time was greater than the time recorded by clinicians without students present. When separated, PT placement data favoured an increase equating to a service offering an average of approximately 25 minutes more time to patients for every hour of direct clinical time. All included papers sampled 5-week block placements, and increases were shown across all student levels, although smaller changes were shown in studies with early-year students. In contrast, the two OT studies did not show this same increase. However, data did not show that OT direct clinical time was reduced with students, which was a concern reported by the majority of academic coordinators and over a third of fieldwork educators in Casares et al. (2003). Interestingly, these OT placements were longer than the PT placements (range 6-12 weeks), but without a larger number of studies, no firm conclusions can be made as to whether differences may be due to placement length, profession or other factors not controlled and/or reported in the studies.

Comparisons of clinical billed units in PT and OT showed variable impacts of students. While units were often not clearly defined within papers, it is presumed these may also be an indicator of available clinical time for patient care. Contrasting results may have been due to heterogeneity in data categorisation, such as variation in placement length or other factors not determined. In addition, despite all data being reported to include student contributions, the specific proportion was not described in most studies and clarification would be beneficial. With the increasing emphasis on charging within healthcare facilities, this result needs to be explored with further research in the studied professions, and investigation in other $\mathrm{AH}$ disciplines may be important.

For healthcare services, particularly those with PT caseloads where relatively more certainty about student impact exists, the likelihood of either maintenance or an overall increase in time available for clinical care should encourage these services to offer student placements. However, more information is needed to better understand the contribution of students and whether this is of clinical benefit, for example, whether patients are receiving high quality care and whether this is more often or for a longer period during student placements. One key contributor to any perceived or actual loss of time may be the time required to teach students (Davies et al., 2011; Ilott, 1996). Unfortunately, the variation in how this was defined in the included papers did not enable any firm conclusions to be made in any subgroup. Some individual papers 
did present the time "lost" in student teaching as offset by other gains, for example, increased available clinical time (Ladyshewsky, 1995). If teaching practices can be well defined, it may be a worthy approach for future research in the studied professions, as well as other $\mathrm{AH}$ disciplines where student teaching time is also of concern, for example, podiatry (Abey, Lea, Callaghan, Shaw, \& Cotton, 2015).

\section{Objective C: The link between clinical time and activity levels}

The link between time used in clinical activities and clinician patient activity levels is productivity, which is important in establishing if any extra time costs could be outweighed by the number of patients seen by a clinician and their student(s). Eight papers reported both time usage and activity measures, enabling some linkage between these two aspects, and four results regarding direct clinical time per patient have been presented. While none of these showed a decrease in direct time per patient, the PT studies did show a significant increase in direct time taken by the supervisor and student(s). Two of these results were from more clinically-complex areas of practice (e.g., burns/plastics), which are often considered more challenging for students to manage as efficiently as a qualified clinician. However, little is known about other plausible factors (e.g., specific supervision practices) that may have also contributed.

A promising direction for those seeking to enhance service productivity using students is reported by Ash, Martin, Rodger, Clark and Graves (2015), who showed a significant increase in individual patients seen when students were present (see Figure 2), without any increase in direct time taken per patient (see Figure 6), supporting the assertion that students could be used to increase service productivity in N\&D. Replication of these results within N\&D and other AH professions would be invaluable and with increased detail regarding supervision practices may provide useful exemplars for clinical services, particularly where increased direct time has been shown.

Interestingly, papers did not report the placement outcome for the student(s), that is, whether they passed or failed, despite this being a factor reported to contribute to a clinician's attitude towards student placements (Davies et al., 2011; Ilott, 1996). The level of clinician experience, both with their clinical caseload as well as with student supervision, was seldom reported, so any potential impact of this factor cannot be determined, nor results easily replicated. Hence, there are clinician, workplace and student factors requiring careful control and/or investigation in future studies.

Although we have reviewed all recent research across similar $\mathrm{AH}$ disciplines and detailed key findings for workplace application, variation and limitations within the studies themselves have reduced our ability to synthesise evidence and make strong conclusions. Specifically, data in both indirect clinical and non-clinical time use, which are important components of $\mathrm{AH}$ work roles, were unable to be further analysed in this paper. The majority of papers in this review were from physiotherapy, which limits the application of the synthesised findings to other professions, particularly nutrition and dietetics, where only one paper was included. In addition, despite the fact data were re-analysed and/or pooled, wherever possible, it is still conceivable that inclusion of unpublished literature may have led to different summary results. It should also 
be noted that while a relevant quality assessment tool was sought, the selected tool also has its own limitations and may not accurately reflect the quality features of the studied papers. In addition, while some papers were excluded due to very low quality, the remaining inclusion of some lower quality research papers with varying outcome measures is a shortfall in our quantitative results. Furthermore, the inclusion criteria may have unnecessarily eliminated some papers or narrowed the geographical regions from which studies were conducted, while including papers from almost 30 years ago may have contributed to the variability given the changes in the healthcare system over time.

While we have suggested that further quantitative research is needed, this data alone will not change student supervision practices in the studied professions, nor other $\mathrm{AH}$ disciplines where these measures are important. There is a need to investigate the link between the quantitative impact of student supervision and the perceptions of all $\mathrm{AH}$ clinicians who manage the complexities of both service provision and facilitating student learning. Hence, we suggest that priorities for further work across AH include:

- strengthening the pool of data regarding clinician time use during periods with and without students

- using data collection methods that highlight individual contributions of students (including aspects where they are not directly supervised) versus supervising clinicians versus their combined input

- linking time data with clinician activity measures during periods with and without students

- Carefully controlling comparison of time use and activity across potential influencing factors such as differing models of supervision, placement length and level of student competence

- linking activity, time use and productivity to clinicians' perceptions of the impact of students.

In all the above categories, we recommend that data collection categories are more clearly defined (particularly in non-clinical categories) and reference measures (e.g., length of a workday) specified to enable better interpretation of each individual study as well as future reviews. Utilising study design and analysis methods based on current higher quality work, such as Rodger et al. (2011), across multiple sites and/or professions, could be beneficial as a starting point. It is also strongly recommended that student supervision models and processes are described in greater detail, so that research can be better applied to relevant settings, particularly if efficiencies are shown.

Finally, this paper has focused on the quantitative impact of students and has not sought to address quality issues that are also important for clinicians and service managers. Services provided by students could be of equal, higher or lesser quality than those delivered by qualified professionals. We note work that has investigated patient satisfaction (e.g., MacDonald et al., 2002) or clinical outcome (e.g., Holland, 1997) in combination with quantitative measurements and recommend that researchers also focus on quality aspects of service provision with students. 


\section{Conclusion}

This review offers important insights into understanding the evidence and gaps in current research regarding the quantitative impact of $\mathrm{AH}$ students on patient activity levels, clinician time use and productivity. There is some evidence that placements have a neutral or positive effect on these measures, however the limitations of individual studies reduce the ability to generalise these findings. More quantitative research is needed to guide health services and universities in embedding innovative and sustainable models of student supervision in the training of the future $\mathrm{AH}$ workforce.

\section{Acknowledgement}

The authors formally thank Dr Jacqueline Raymond for her invaluable advice during study design, analysis and manuscript preparation.

\section{Funding and conflicts of interest}

None

\section{References}

Abey, S., Lea, S., Callaghan, L., Shaw, S., \& Cotton, D. (2015). Identifying factors which enhance capacity to engage in clinical education among podiatry practitioners: An action research project. Journal of Foot and Ankle Research, 8, 66. doi:10.1186/s13047-015-0123-4

Ash, S., Martin, E. K., Rodger, S., Clark, M., \& Graves, N. (2015). Student and supervisor productivity change during nutrition and dietetic practice placements: A cohort study. Nutrition \& Dietetics, 72 (2), 163-169. doi:10.1111/17470080.12093

Borenstein, M., Hegdes, L. V., Higgins, J. P. T., \& Rothstein, H. R. (2009). Introduction to meta-analysis. Chichester, UK: John Wiley \& Sons.

Borenstein, M., Hedges, L., Higgins, J. P. T., \& Rothstein, H. R. (2014). Comprehensive Meta Analysis Version 3.0. Englewood, NJ: BioStat.

Borenstein, M., Higgins, J. P. T., Hedges, L. V., \& Rothstein, H. R. (2017). Basics of meta-analysis: $\mathrm{I}^{2}$ is not an absolute measure of heterogeneity. Research Synthesis Methods, 8(1), 5-18. doi:10.1002/jrsm.1230

Bowles, K., Haines, T. P., Molloy, E., Kent, F., Sevenhuysen, S., \& Tai, J. (2014). The costs and benefits of providing undergraduate student clinical placements for a health service organisation: A rapid review. Retrieved from https://www.saxinstitute.org.au/ wp-content/uploads/The-costs-and-benefits-of-providing-undergraduate-studentclinical-place....pdf

Briffa, C., \& Porter, J. (2013). A systematic review of the collaborative clinical education model to inform speech-language pathology practice. International Journal of Speech-Language Pathology, 15(6), 564-574. doi:10.3109/17549507.20 13.763290 
Bristow, D., \& Hagler, P. (1994). Impact of physical therapy students on patient service delivery and professional staff time. Physiotherapy Canada, 46(4), 275-280.

Bristow, D., \& Hagler, P. (1997). Comparison of individual physical therapists' productivity to that of combined physical therapist-student pairs. Physiotherapy Canada, 49(1), 16-23.

Campbell, N., Eley, D. S., \& McAllister, L. (2014). Investigating personality and conceptualising allied health as person or technique oriented. Australian Health Review, 38(1), 86-92. doi:10.1071/ah13109

Casares, G. S., Bradley, K. P., Jaffe, L. E., \& Lee, G. P. (2003). Impact of the changing health care environment on fieldwork education: Perceptions of occupational therapy educators. Journal of Allied Health, 32(4), 246-251.

Coulson, E., Woekel, D., Copenhauer, R., Gallatin, T., Hawkins, J., Hixson, L., \& Pruim, C. (1991). Effects of clinical education on the productivity of private practice facilities. Journal of Physical Therapy Education, 5(1), 29-32.

Davies, R., Hanna, E., \& Cott, C. (2011). “They put you on your toes”: Physical therapists' perceived benefits from and barriers to supervising students in the clinical setting. Physiotherapy Canada, 63(2), 224-233.

Dean, C. M., Alam, M., \& Refshauge, K. (2010). Clinical training requirements for the Faculty of Health Sciences. Synergy, 30, 22-27.

Dillon, L. S., Tomaka, J. W., Chriss, C. E., Gutierrez, C. P., \& Hairston, J. M. (2003). The effect of student clinical experiences on clinician productivity. Journal of Allied Health, 32(4), 261-265.

Dupont, L., Gauthier-Gagnon, C., Roy, R., \& Lamoureux, M. (1997). Group supervision and productivity: From myth to reality. Journal of Physical Therapy Education, 11(2), 31-37.

Evenson, M. E., Roberts, M., Kaldenberg, J., Barnes, M. A., \& Ozelie, R. (2015). National Survey of Fieldwork Educators: Implications for occupational therapy education. American Journal of Occupational Therapy, 69(Suppl. 2), 1-5. doi:10.5014/ajot.2015.019265

Graham, C. L., Catlin, P., Morgan, J., \& Martin, E. (1991). Comparison of 1-dayper-week, 1-week, and 5-week clinical education experiences. Journal of Physical Therapy Education, 5(1), 18-23.

Hall, M., Poth, C., Manns, P., \& Beaupre, L. (2015). To supervise or not to supervise a physical therapist student: A national survey of Canadian physical therapists. Journal of Physical Therapy Education, 29(3), 58-67.

Hancock, J. J. (1997). Impact of speech-language pathology students on patient care. Unpublished master's thesis, University of Alberta, Edmonton, Alberta, Canada.

Hancock, J., \& Hagler, P. (1998). A pilot study of the effects of SL-P practicum students on service delivery. Journal of Speech-Language Pathology and Audiology, 22(3), 141-150. 
Health Workforce Australia (HWA). (2014). Australia's health workforce series: Speech pathologists in focus. Retrieved from https://industry.gov.au/Office-of-theChief-Economist/SkilledOccupationList/Documents/2015Submissions/SpeechPathology-Australia.pdf

Holland, K. A. (1997). Does taking students increase your waiting lists? Physiotherapy, 83(4), 166-172.

Huddleston, R. (1999). Clinical placements for the professions allied to medicine, part 2: Placement shortages? Two models that can solve the problem. The British Journal of Occupational Therapy, 62(7), 295-298.

Ilott, I. (1996). Ranking the problems of fieldwork supervision reveals a new problem: Failing students. The British Journal of Occupational Therapy, 59(11), 525-528. doi:10.1177/030802269605901110

Ladyshewsky, R. K. (1995). Enhancing service productivity in acute care inpatient settings using a collaborative clinical education model. Physical Therapy, 75(6), 503-510.

Ladyshewsky, R. K., Barrie, S. C., \& Drake, V. M. (1998). A comparison of productivity and learning outcome in individual and cooperative physical therapy clinical education models. Physical Therapy, 78(12), 1288-1298.

Ladyshewsky, R., Bird, N., \& Finney, J. (1994). The impact on departmental productivity during physical therapy student placements: An investigation of outpatient physical therapy services. Physiotherapy Canada, 46(2), 89-93.

Law, M., Stewart, D., Pollock, N., Letts, L., Bosch, J., \& Westmorland, M. (2014). Guidelines for critical review form: Quantitative studies. Retrieved from https://srsmcmaster.ca/wp-content/uploads/2015/04/Guidelines-for-Critical-Review-FormQuantiative-Studies-English.pdf

Law, M., Stewart, D., Pollock, N., Letts, L., Bosch, J., Westmorland, M., \& Philpot, A. (1998). Evidence-based practice research group. Retrieved from https://srsmcmaster.ca/research/evidence-based-practice-research-group/

Lekkas, P., Larsen, T., Kumar, S., Grimmer, K., Nyland, L., Chipchase, L., . . . Finch, J. (2007). No model of clinical education for physiotherapy students is superior to another: A systematic review. Australian Journal of Physiotherapy, 53(1), 19-28.

Liberati, A., Altman, D. G., Tetzlaff, J., Mulrow, C., Gøtzsche, P. C., Ioannidis, J. P. A., . . Moher, D. (2009). The PRISMA statement for reporting systematic reviews and meta-analyses of studies that evaluate health care interventions: Explanation and elaboration. PLOS Medicine, 6(7), e1000100. doi:10.1371/ journal.pmed.1000100

Lindeblad, S. K. (1998). Comparison of two clinical education models on the functioning of three acute care physical therapy departments. Unpublished doctoral dissertation, University of Miami, Coral Gables, Florida.

Loewen, P., Legal, M., Gamble, A., Shah, K., Tkachuk, S., \& Zed, P. (2017). Learner: preceptor ratios for practice-based learning across health disciplines: A systematic review. Medical Education, 51(2), 146-157. doi:10.1111/medu.13144 
MacDonald, C. A., Cox, P. D., \& Bartlett, D. J. (2002). Productivity and client satisfaction: A comparison between physical therapists and student-therapist pairs. Physiotherapy Canada, 54(2), 92-101.

McAllister, L. (2005). Issues and innovations in clinical education. International Journal of Speech-Language Pathology, 7(3), 138-148. doi:10.1080/14417040500181239

Moher, D., Liberati, A., Tetzlaff, J., Altman, D. G., \& The, P. G. (2009). Preferred reporting items for systematic reviews and meta-analyses: The PRISMA statement. PLOS Medicine, 6(7), e1000097. doi:10.1371/journal.pmed.1000097

Moore, J. H., Glenesk, K. T., Hulsizer, D. K., McCright, B. E., Wrenn, C., \& Sander, T., .. . Childs, J. D. (2014). Impact of an innovative clinical internship model in the US Army-Baylor Doctoral Program in physical therapy. The United States Army Medical Department Journal, Jan-Mar, 30-34.

National Health Workforce Taskforce. (2009). Health workforce in Australia and factors for current shortages. Retrieved from http://webarchive.nla.gov.au/ gov/20160226234255/http://www.ahwo.gov.au/documents/NHWT/The\%20 health\%20workforce $\% 20 \mathrm{in} \% 20$ Australia\%20and $\% 20$ factors $\% 20$ influencing $\% 20$ current $\% 20$ shortages.pdf

O'Brien, C. W., Anderson, R., Ayzenberg, B., Chute, P., Farnsworth, T., McLaughlin, R., . . Maillet, J. O. S. (2017). Employers' viewpoint on clinical education. Journal of Allied Health, 46(3), 131-137.

Ozelie, R., Janow, J., Kreutz, C., Mulry, M. K., \& Penkala, A. (2015). Supervision of occupational therapy level II fieldwork students: Impact on and predictors of clinician productivity. American Journal of Occupational Therapy, 69(1), 1-7. doi:10.5014/ajot.2015.013532

Pabian, P. S., Dyson, J., \& Levine, C. (2017). Physical therapist productivity using a collaborative clinical education model within an acute care setting: A longitudinal study. Journal of Physical Therapy Education, 31(2), 11-17.

Paterson, M. L. (1997). Clinician productivity with and without students. The Occupational Therapy Journal of Research, 17(1), 48-54.

Phelan, S. E., Daniels, M. G., \& Hewitt, L. (1999). The costs and benefits of clinical education. Laboratory Medicine, 30(11), 714-720.

Pivko, S. E., Abbruzzese, L. D., Duttaroy, P., Hansen, R. L., \& Ryans, K. (2016). Effect of physical therapy students' clinical experiences on clinician productivity. Journal of Allied Health, 45(1), 33-40.

Pretorius, A., Karunaratne, N., \& Fehring, S. (2016). Australian physiotherapy workforce at a glance: A narrative review. Australian Health Review, 40(4), 438442. doi:10.1071/AH15114

Rindflesch, A. B., Dunfee, H. J., Cieslak, K. R., Eischen, S. L., Trenary, T., Calley, D. Q., \& Heinle, D. K. (2009). Collaborative model of clinical education in physical and occupational therapy at the Mayo Clinic. Journal of Allied Health, 38(3), 132-142. 
Roberts, M., Evenson, M. E., Kaldenberg, J., Barnes, M. A., \& Ozelie, R. (2015, May 25). Fieldwork education survey: Demand for innovative and creative solutions. OT Practice, 15-16. Retrieved from https://www.aota.org/Publications-News/otp/ Archive/2015.aspx

Rodger, S., Stephens, E., Clark, M., Ash, S., \& Graves, N. (2011). Occupational therapy students' contribution to occasions of service during practice placements in health settings. Australian Occupational Therapy Journal, 58(6), 412-418.

Rodger, S., Stephens, E., Clark, M., Ash, S., Hurst, C., \& Graves, N. (2012). Productivity and time use during occupational therapy and nutrition/dietetics clinical education: A cohort study. PLoS ONE, 7(8), e44356. doi:10.1371/journal. pone. 0044356

Rodger, S., Webb, G., Devitt, L., Gilbert, J., Wrightson, J., \& McMeeken, J. (2008). Clinical education and practice placements in the allied health professions: An international perspective. Journal of Allied Health, 37(1), 53-62.

Schlosser, R. W., Wendt, O., \& Sigafoos, J. (2007). Not all systematic reviews are created equal: Considerations for appraisal. Evidence-Based Communication Assessment and Intervention, 1(3), 138-150. doi:10.1080/17489530701560831

Sevenhuysen, S. L., \& Haines, T. (2011). The slave of duty: Why clinical educators across the continuum of care provide clinical education in physiotherapy. Hong Kong Physiotherapy Journal, 29(2), 64-70.

Short, K., Lincoln, M., \& Gilsenan, K. (2001). The evaluation of the impact of student placements on a large area health service. Paper presented at the National Conference of the Speech Pathology Association of Australia, Melbourne, Victoria, Australia.

Sterne, J. A. C., Sutton, A. J., Ioannidis, J. P. A., Terrin, N., Jones, D. R., Lau, J., . . . Higgins, J. P. T. (2011). Recommendations for examining and interpreting funnel plot asymmetry in meta-analyses of randomised controlled trials. BMJ, 343(7818), 302-307. doi:10.1136/bmj.d4002

United States Department of Labor (2018). Occupational outlook handbook. Retrieved from http://www.bls.gov/ooh/

Valentine, J. C., Pigott, T. D., \& Rothstein, H. R. (2010). How many studies do you need? A Primer on statistical power for meta-analysis. Journal of Educational and Behavioral, 35(3), 375. 